\title{
A NONCONFORMING MIXED MULTIGRID METHOD FOR THE PURE TRACTION PROBLEM IN PLANAR LINEAR ELASTICITY
}

\author{
SUSANNE C. BRENNER
}

\begin{abstract}
A robust optimal-order multigrid method for the pure traction problem in two-dimensional linear elasticity is studied. The finite element discretization is a variant of a mixed method proposed by Falk where the displacement is approximated by nonconforming piecewise linear functions and the "pressure" is approximated by piecewise constant functions on a coarser grid. Full multigrid convergence is obtained. The performance of this multigrid algorithm does not deteriorate as the material becomes nearly incompressible.
\end{abstract}

\section{INTRODUCTION}

Let $\Omega$ be a bounded convex polygonal domain in $\mathbb{R}^{2}$. The pure traction boundary value problem for planar linear elasticity is given by

$$
-\operatorname{div}\{2 \mu \underset{\sim}{\epsilon}(\underset{\sim}{u})+\lambda \operatorname{tr} \underset{\sim}{\epsilon}(\underset{\sim}{\mathcal{u}})) \underset{\sim}{\delta}\}=\underset{\sim}{f} \text { in } \Omega,
$$

$$
(2 \mu \underset{\sim}{\epsilon}(\underset{\sim}{u})+\lambda \operatorname{tr}(\underset{\sim}{\epsilon}(\underset{\sim}{u})) \underset{\sim}{\delta}) \underset{\sim}{\nu}=\underset{\sim}{g} \quad \text { on } \partial \Omega,
$$

where $\underset{\sim}{u}$ is the displacement, $\underset{\sim}{f}$ is the body force, $\underset{\sim}{g}$ is the boundary traction, $\mu>0, \lambda>0$ are the Lamé constants, and $\underset{\sim}{\nu}$ is the unit outer normal. Throughout this paper, an undertilde is used to denote vector-valued functions, operators and their associated spaces. Double undertildes are used for matrixvalued functions and operators. We define

$$
\begin{gathered}
\underset{\sim}{\operatorname{grad} p}=\left(\begin{array}{c}
\partial p / \partial x_{1} \\
\partial p / \partial x_{2}
\end{array}\right), \quad \underset{\sim}{\operatorname{div}} \underset{\sim}{\tau}=\left(\begin{array}{l}
\partial \tau_{11} / \partial x_{1}+\partial \tau_{12} / \partial x_{2} \\
\partial \tau_{21} / \partial x_{1}+\partial \tau_{22} / \partial x_{2}
\end{array}\right), \\
\operatorname{curl} p=\left(\begin{array}{c}
\partial p / \partial x_{2} \\
-\partial p / \partial x_{1}
\end{array}\right), \\
\operatorname{div} \underset{\sim}{v}=\partial v_{1} / \partial x_{1}+\partial v_{2} / \partial x_{2}, \quad \operatorname{rot} \underset{\sim}{v}=-\partial v_{1} / \partial x_{2}+\partial v_{2} / \partial x_{1},
\end{gathered}
$$

Received by the editor January 13, 1993 and, in revised form, December 17, 1993.

1991 Mathematics Subject Classification. Primary 65F10, 65N30, 65N55.

Key words and phrases. Linear elasticity, mixed finite element, multigrid, nonconforming, traction problem.

This work was supported in part by the National Science Foundation under Grant Nos. DMS90-96126 and DMS-92-09332. 
and

$$
\underset{\approx}{\operatorname{grad}} \underset{\sim}{v}=\left(\begin{array}{ll}
\partial v_{1} / \partial x_{1} & \partial v_{1} / \partial x_{2} \\
\partial v_{2} / \partial x_{1} & \partial v_{2} / \partial x_{2}
\end{array}\right)
$$

We also define

$$
\underset{\approx}{\delta}=\left(\begin{array}{ll}
1 & 0 \\
0 & 1
\end{array}\right), \quad \underset{\approx}{\chi}=\left(\begin{array}{rr}
0 & -1 \\
1 & 0
\end{array}\right)
$$

and

$$
\operatorname{tr}(\underset{\approx}{\tau})=\underset{\approx}{\tau}: \underset{\approx}{\delta}
$$

where

$$
\underset{\approx}{\sigma}: \underset{\approx}{\tau}=\sum_{i=1}^{2} \sum_{j=1}^{2} \sigma_{i j} \tau_{i j}
$$

Finally,

$$
\underset{\approx}{\epsilon}(\underset{\sim}{v})=\frac{1}{2}\left[\underset{\approx}{\operatorname{grad}} \underset{\sim}{v}+(\underset{\approx}{\operatorname{grad}} \underset{\sim}{v})^{t}\right] .
$$

We assume that the Lamé constants $(\mu, \lambda)$ belong to the range $\left[\mu_{1}, \mu_{2}\right] \times$ $\left[\lambda_{1}, \infty\right)$, where $\mu_{1}, \mu_{2}, \lambda_{1}$ are three fixed positive constants.

We shall denote by ${\underset{\sim}{H}}^{k}(\Omega)$ the standard $L^{2}$ Sobolev spaces of vector-valued functions, and we use the following conventions for Sobolev norms and seminorms:

$$
\begin{aligned}
& \|\underset{\sim}{\underset{\sim}{v}}\|_{\mathcal{\sim}^{m}(\Omega)}:=\left(\int_{\Omega} \sum_{|\alpha| \leq m}\left|\partial^{\alpha} \underset{\sim}{\underset{\sim}{v}}\right|^{2} d x\right)^{1 / 2} \text { and } \\
& |\underset{\sim}{v}|_{\mathcal{\sim}^{m}(\Omega)}:=\left(\int_{\Omega} \sum_{|\alpha|=m}\left|\partial^{\alpha} \underset{\sim}{v}\right|^{2} d x\right)^{1 / 2} .
\end{aligned}
$$

Let $\mathbf{R M}=\left\{\underset{\sim}{v}:{\underset{\sim}{v}}^{t}=\left(a+b x_{2}, c-b x_{1}\right), a, b, c \in \mathbb{R}\right\}$ be the space of infinitesimal rigid motions. Since $\underset{\approx}{\epsilon}(\underset{\sim}{v})=\underset{\approx}{0}$ for all $\underset{\sim}{v} \in \mathbf{R M}$, one should look for a unique solution of $(1.1)$ in a subspace of ${\underset{\sim}{H}}^{k}(\Omega)$ which is transversal to $\mathrm{RM}$. The unique solvability and regularity theorems are usually stated on the space

$$
\stackrel{\sim}{\sim}^{k}(\Omega)=\left\{\underset{\sim}{v} \in{\underset{\sim}{H}}^{k}(\Omega): \int_{\Omega} \underset{\sim}{v} d x=\underset{\sim}{0}, \int_{\Omega} \operatorname{rot} \underset{\sim}{v} d x=0\right\} .
$$

However, for the design and analysis of a multigrid method, it is better to use the space

$$
{\underset{\sim}{H}}_{\perp}^{k}(\Omega)=\left\{\underset{\sim}{v} \in{\underset{\sim}{H}}^{k}(\Omega): \int_{\Omega} \underset{\sim}{v} \cdot \underset{\sim}{w} d x=0 \quad \forall \underset{\sim}{w} \in \underset{\sim}{\mathbf{R}}\right\} .
$$

We also use the notation $\underset{\sim}{L_{\perp}^{2}}$ for $\underset{\sim}{H_{\perp}}{ }_{\perp}$. We assume that the origin of our coordinate system is chosen to be the centroid of $\Omega$, so that $\mathrm{RM}$ has the following orthonormal basis:

$$
{\underset{\sim}{\psi}}_{1}=\frac{1}{|\Omega|^{1 / 2}}\left(\begin{array}{l}
1 \\
0
\end{array}\right), \quad{\underset{\sim}{2}}_{2}=\frac{1}{|\Omega|^{1 / 2}}\left(\begin{array}{l}
0 \\
1
\end{array}\right), \quad \underset{\sim}{\psi_{3}}=\frac{1}{\omega}\left(\begin{array}{r}
-x_{2} \\
x_{1}
\end{array}\right)
$$

where $\omega=\sqrt{\int_{\Omega}\left(x_{1}^{2}+x_{2}^{2}\right) d x}$. 
Let the operators $T_{\perp}^{\wedge}$ and $T_{\wedge}^{\perp}$ be defined by

$$
T_{\perp}^{\wedge}(\underset{\sim}{v})=\underset{\sim}{v}-\frac{\omega}{2|\Omega|}\left(\int_{\Omega} \operatorname{rot} \underset{\sim}{v} d x\right) \underset{\sim}{\psi}
$$

and

$$
T_{\wedge}^{\perp}(\underset{\sim}{v})=\underset{\sim}{v}-\left(\int_{\Omega} \underset{\sim}{v} \cdot \underset{\sim}{\psi} d x\right) \underset{\sim}{\psi_{3}}
$$

Then clearly for $k \geq 1$,

$$
\begin{gathered}
T_{\perp}^{\wedge}:{\underset{\sim}{H}}_{\perp}^{k}(\Omega) \longrightarrow{\underset{\sim}{\hat{H}}}^{k}(\Omega) \text { and } T_{\wedge}^{\perp}:{\underset{\sim}{\hat{H}}}^{k}(\Omega) \longrightarrow{\underset{\sim}{H}}_{\perp}^{k}(\Omega), \\
T_{\wedge}^{\perp}\left(T_{\perp}^{\wedge} \underset{\sim}{v}\right)=\underset{\sim}{v} \quad \forall \underset{\sim}{v} \in{\underset{\sim}{H}}_{\perp}^{k}(\Omega) \text { and } \\
T_{\perp}^{\wedge}\left(T_{\wedge}^{\perp} \underset{\sim}{v}\right)=\underset{\sim}{v} \quad \forall \underset{\sim}{v} \in{\underset{\sim}{\hat{H}}}^{k}(\Omega), \\
\underset{\sim}{\epsilon}(\underset{\sim}{v})=\underset{\sim}{\epsilon}\left(T_{\perp}^{\wedge}(\underset{\sim}{v})\right) \quad \forall \underset{\sim}{v} \in{\underset{\sim}{H}}_{\perp}^{k}(\Omega),
\end{gathered}
$$

and

$$
\operatorname{div}(\underset{\sim}{v})=\operatorname{div}\left(T_{\perp}^{\wedge}(\underset{\sim}{v})\right) \quad \forall \underset{\sim}{v} \in{\underset{\sim}{H}}_{\perp}^{k}(\Omega) .
$$

There exist positive constants $C_{1}$ and $C_{2}$ such that $(k \geq 1)$

$$
C_{1}\left\|T_{\perp}^{\wedge}(\underset{\sim}{v})\right\|_{\sim^{H}(\Omega)}{ }^{k}\left(\|\underset{\sim}{v}\|_{\sim^{H}(\Omega)} \leq C_{2}\left\|T_{\perp}^{\wedge}(\underset{\sim}{v})\right\|_{\sim^{H}(\Omega)}{ }^{k}\left(\underset{\sim}{v} \in{\underset{\sim}{H}}_{\perp}^{k}(\Omega) .\right.\right.
$$

By Friedrichs' inequality (cf. [9, Theorem 1.1.5]), (1.13) also holds for $|\cdot|_{\sim}^{\sim^{\ell}(\Omega)}$ $(1 \leq \ell \leq k)$ norms:

$$
C_{1}\left|T_{\perp}^{\wedge}(\underset{\sim}{v})\right|_{\mathcal{\sim}^{\ell}(\Omega)} \leq|\underset{\sim}{v}|_{\underset{\sim}{H^{\ell}(\Omega)}} \leq C_{2}\left|T_{\perp}^{\wedge}(\underset{\sim}{v})\right|_{\sim^{\prime}(\Omega)} \quad \forall \underset{\sim}{v} \in \underset{\sim}{H_{\perp}^{k}}(\Omega) .
$$

Using these operators, one can translate results for the $\underset{\sim}{\hat{H}}{ }^{k}(\Omega)$-space to the $\stackrel{H}{\sim}_{\perp}^{k}(\Omega)$-space and vice versa.

Since the boundary of a polygon has corners, the boundary conditions in (1.1) must be carefully interpreted. We shall denote by $S_{i}, 1 \leq i \leq n$, the vertices of $\Omega$; by $\Gamma_{i}, 1 \leq i \leq n$, the open line segments joining $S_{i}$ to $S_{i+1}$; by $\underset{\sim}{\tau}$ the positively oriented unit tangent along $\Gamma_{i}$; and by $\nu_{i}$ the unit outer normal along $\Gamma_{i}$. Henceforth, $S_{n+1}$ and $\Gamma_{n+1}$ should be interpreted as $S_{1}$ and $\Gamma_{1}$, respectively.

Let $p \in H^{1 / 2}\left(\Gamma_{i}\right)$ and $q \in H^{1 / 2}\left(\Gamma_{i+1}\right)$. We say that $p \equiv q$ at $S_{i+1}$ if $\int_{0}^{\delta}|q(s)-p(-s)|^{2} \frac{d s}{s}<\infty$, where $s$ is the oriented arc length measured from $S_{i+1}$, and $\delta$ is a positive number less than $\min \left\{\left|\Gamma_{i}\right|: 1 \leq i \leq n\right\}$.

Equation (1.1) can be written more precisely as

$$
\begin{aligned}
&-\operatorname{div}\{2 \mu \underset{\sim}{\epsilon}(\underset{\sim}{u})+\lambda \operatorname{tr}(\underset{\sim}{\epsilon}(\underset{\sim}{)})) \underset{\approx}{\delta}\}=\underset{\sim}{f} \text { in } \Omega, \\
&(2 \mu \underset{\sim}{\epsilon}(\underset{\sim}{\mathcal{u}})+\lambda \operatorname{tr}(\underset{\sim}{\epsilon})) \underset{\sim}{\delta}) \underset{\sim}{\nu_{i}} \mid \Gamma_{i}={\underset{\sim}{g}}_{i}, \quad 1 \leq i \leq n,
\end{aligned}
$$

where $\underset{\sim}{f} \in \underset{\sim}{L}{ }^{2}(\Omega)$, and $\underset{\sim}{g} \in \underset{\sim}{H}{ }^{1 / 2}\left(\Gamma_{i}\right)$ satisfy

$$
{\underset{\sim}{g}}_{i} \cdot \stackrel{\nu}{\sim}_{i+1} \equiv{\underset{\sim}{g}}_{i+1} \cdot \stackrel{\nu}{\sim}_{i} \text { at } S_{i+1} \text { for } 1 \leq i \leq n .
$$


If we assume that the following compatibility condition holds,

$$
\int_{\Omega} \underset{\sim}{f} \cdot \underset{\sim}{v} d x+\left.\sum_{i=1}^{n} \int_{\Gamma_{i}} \underset{\sim}{g} \cdot \underset{\sim}{v}\right|_{\Gamma_{i}} d s=0 \quad \forall \underset{\sim}{v} \in \underset{\sim}{\mathrm{RM}}
$$

then the pure traction boundary value problem (1.15) has a unique solution $\underset{\sim}{\hat{u}} \in{\underset{\sim}{\hat{H}}}^{2}(\Omega)$. Moreover, there exists a positive constant $C_{\Omega}$ such that

$$
\|\underset{\sim}{\hat{u}}\|_{\underset{\sim}{H^{2}(\Omega)}}+\lambda\|\operatorname{div} \underset{\sim}{\hat{u}}\|_{H^{1}(\Omega)} \leq C_{\Omega}\left\{\|\underset{\sim}{f}\|_{\underset{\sim}{L^{2}(\Omega)}}+\sum_{i=1}^{n}\|\underset{\sim}{g}\|_{\underset{\sim}{H}}{ }^{1 / 2\left(\Gamma_{i}\right)}\right\}
$$

(cf. $[3,7,8]$ ).

By using (1.10) and (1.11), we see that (1.15) also has a unique solution ${\underset{\sim}{u}}_{\perp} \in{\underset{\sim}{H}}_{\perp}^{2}(\Omega)$ and

$$
\stackrel{\hat{u}}{\sim}=T_{\perp}^{\wedge} \stackrel{\sim}{\sim}_{\perp}
$$

Therefore, (1.12), (1.13), and (1.18) imply that there exists a positive constant $C_{\Omega}^{\prime}$ such that

(1.20) $\|\underset{\sim}{u}\|_{{\underset{\sim}{H}}^{2}(\Omega)}+\lambda\|\operatorname{div} \underset{\sim}{u}\|_{H^{1}(\Omega)} \leq C_{\Omega}^{\prime}\left\{\|\underset{\sim}{f}\|_{\sim^{2}(\Omega)}+\sum_{i=1}^{n}\|\underset{\sim}{g}\|_{H_{\sim}^{1 / 2}\left(\Gamma_{i}\right)}\right\}$.

From now on, we will simply denote $\underset{\sim}{\mathcal{u}}$ by $\underset{\sim}{\mathcal{u}}$.

Henceforth, (1.16) and (1.17) are assumed to be true. Let $\gamma=\lambda /(2 \mu)$ and $p=\gamma \operatorname{div} \underset{\sim}{u}$; then a mixed formulation for $(1.15)$ is:

Find $(\underset{\sim}{\sim}, p) \in{\underset{\sim}{H}}_{\perp}^{1}(\Omega) \times L^{2}(\Omega)$ such that

$$
\begin{aligned}
& \left.\int_{\Omega} \underset{\approx}{\epsilon}(\underset{\sim}{u}): \underset{\approx}{\epsilon} \underset{\sim}{v}\right) d x+\int_{\Omega} p(\operatorname{div} \underset{\sim}{v}) d x \\
& =\frac{1}{2 \mu}\left[\int_{\Omega} \underset{\sim}{f} \cdot \underset{\sim}{v} d x+\left.\sum_{i=1}^{n} \int_{\Gamma_{i}} \underset{\sim}{g} \cdot \underset{\sim}{v}\right|_{\Gamma_{i}} d s\right], \\
& \int_{\Omega}(\operatorname{div} \underset{\sim}{u}) q d x-\frac{1}{\gamma} \int_{\Omega} p q d x=0
\end{aligned}
$$

for all $(\underset{\sim}{v}, q) \in \underset{\sim}{H}{ }_{\perp}^{1}(\Omega) \times L^{2}(\Omega)$.

Equation (1.21) can be written concisely as

$$
\mathscr{B}((\underset{\sim}{u}, p),(\underset{\sim}{v}, q))=\frac{1}{2 \mu}\left[\int_{\Omega} \underset{\sim}{f} \cdot \underset{\sim}{v} d x+\left.\sum_{i=1}^{n} \int_{\Gamma_{i}} \underset{\sim}{g} \underset{i}{*} \underset{\sim}{v}\right|_{\Gamma_{i}} d s\right]
$$

for all $(\underset{\sim}{v}, q) \in{\underset{\sim}{H}}_{\perp}^{1}(\Omega) \times L^{2}(\Omega)$, where the symmetric bilinear form $\mathscr{B}(\cdot, \cdot)$ on $\underset{\sim}{H}{ }^{1}(\Omega) \times L^{2}(\Omega)$ is defined by

$$
\begin{aligned}
& \mathscr{B}\left(\left({\underset{\sim}{1}}_{1}^{v}, q_{1}\right),\left(\underset{\sim}{v}, q_{2}\right)\right) \\
& \quad=\int_{\Omega}\left\{\underset{\approx}{\epsilon}(\underset{\sim 1}{v}): \underset{\approx}{\epsilon}(\underset{\sim}{v})+q_{1}(\operatorname{div} \underset{\sim}{v})+(\operatorname{div} \underset{\sim 1}{v}) q_{2}-\frac{1}{\gamma} q_{1} q_{2}\right\} d x .
\end{aligned}
$$


It is clear from the definition of $\mathscr{B}$ that

$$
\begin{aligned}
& \left|\mathscr{B}\left(\left({\underset{\sim}{v}}_{1}, q_{1}\right),\left(\underset{\sim}{v}, q_{2}\right)\right)\right| \leq \max \left(\sqrt{2}, \frac{2 \mu_{2}}{\lambda_{1}}\right)\left(|\underset{\sim}{v}|_{\sim^{1}(\Omega)}+\left\|q_{1}\right\|_{L^{2}(\Omega)}\right) \\
& \cdot\left(|\underset{\sim 2}{\underset{\sim}{v}}|_{H^{1}(\Omega)}+\left\|q_{2}\right\|_{L^{2}(\Omega)}\right) \text {. }
\end{aligned}
$$

Let $\mathscr{T}_{k}(k \geq 0)$ be a sequence of triangulations of $\Omega$, where $\mathscr{T}_{k+1}$ is obtained by connecting the midpoints of each of the triangles in $\mathscr{T}_{k}$. We will denote $\max \left\{\operatorname{diam} T: T \in \mathscr{T}_{k}\right\}$ by $h_{k}$. For $k \geq 0$, let $Q_{k}=\left\{q: q \in L^{2}(\Omega)\right.$ and $\left.q\right|_{T}$ is a constant for all $\left.T \in \mathscr{T}_{k}\right\}$. The nonconforming finite element spaces $\sim_{k}(k \geq 1)$ are defined as follows:

$$
\begin{array}{r}
\underset{\sim}{V}=\left\{\underset{\sim}{v}: \underset{\sim}{v} \in \underset{\sim}{L^{2}}(\Omega),\left.\underset{\sim}{v}\right|_{T} \text { is linear for all } T \in \mathscr{T}_{k}, \underset{\sim}{v}\right. \text { is continuous } \\
\text { at the midpoints of interelement boundaries }\} .
\end{array}
$$

Since $\underset{\sim}{V}$ is nonconforming, any differentiation of members of $\underset{\sim}{V}$ must be done piecewise. We define the operators $\operatorname{div}_{k}, \operatorname{rot}_{k}$, and $\underset{\approx}{\operatorname{grad}_{k}}$ as follows:

$$
\begin{gathered}
\left.\left(\operatorname{div}_{k} \underset{\sim}{v}\right)\right|_{T}=\operatorname{div}\left(\left.\underset{\sim}{v}\right|_{T}\right), \\
\left.\left(\operatorname{rot}_{k} \underset{\sim}{v}\right)\right|_{T}=\operatorname{rot}\left(\left.\underset{\sim}{v}\right|_{T}\right), \\
\left(\underset{\approx}{\left.\operatorname{grad}_{k} \underset{\sim}{v}\right)\left.\right|_{T}}=\underset{\approx}{\operatorname{grad}}\left(\left.\underset{\sim}{v}\right|_{T}\right),\right.
\end{gathered}
$$

for all $T \in \mathscr{T}_{k}$. We also denote by $\underset{\sim}{V} \underset{k}{\perp}$ the subspaces of $\underset{\sim}{V}$ whose members satisfy

$$
\int_{\Omega} \underset{\sim}{v} \cdot \underset{\sim}{w} d x=0 \quad \forall \underset{\sim}{w} \in \underset{\sim}{\mathbf{R M}}
$$

The following discretization of (1.22) is a modification of one introduced by Falk in [6].

Find $\left(\underset{\sim}{u}, p_{k}\right) \in \underset{\sim}{V} \underset{k}{\perp} \times Q_{k-1}(k \geq 1)$ such that

$$
\mathscr{B}_{k}\left(\left({\underset{\sim}{u}}_{k}, p_{k}\right),(\underset{\sim}{v}, q)\right)=\frac{1}{2 \mu}\left[\int_{\Omega} \underset{\sim}{f} \cdot \underset{\sim}{v} d x+\sum_{i=1}^{n} \int_{\Gamma_{i}} \underset{\sim}{g} \cdot \underset{\sim}{v} \mid \Gamma_{i} d s\right]
$$

for all $(\underset{\sim}{v}, q) \in \underset{\sim}{V} \underset{k}{\perp} \times Q_{k-1}$. Here the symmetric bilinear form $\mathscr{B}_{k}$ on $\left(\underset{\sim}{H^{1}}(\Omega)+\underset{\sim}{V}\right) \times L^{2}(\Omega)$ is defined by

$$
\begin{aligned}
& \mathscr{B}_{k}\left(\left({\underset{\sim}{v}}_{1}, q_{1}\right),\left({\underset{\sim}{v}}_{2}, q_{2}\right)\right) \\
& =\int_{\Omega}\left\{\underset{\approx k}{\epsilon_{\sim}^{*}}(\underset{\sim}{v}): \underset{\approx k}{{\underset{\approx}{*}}_{*}^{*}}\left({\underset{\sim}{v}}_{2}\right)+q_{1}\left(\operatorname{div}_{k} \underset{\sim 2}{v}\right)+\left(\operatorname{div}_{k} \underset{\sim 1}{v_{1}}\right) q_{2}-\frac{1}{\gamma} q_{1} q_{2}\right\} d x,
\end{aligned}
$$

where

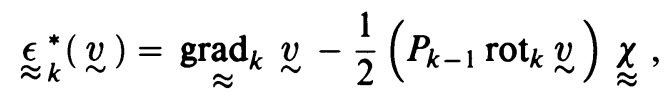


and $P_{k}(k \geq 0)$ is the $L^{2}$ orthogonal projection onto $Q_{k}$. Note that there exists a positive constant $C$ such that

$$
\begin{aligned}
& \left|\mathscr{B}_{k}\left(\left(\underset{\sim}{\underset{\sim}{v}}, q_{1}\right),\left(\underset{\sim}{\underset{\sim}{v}}, q_{2}\right)\right)\right| \\
& \quad \leq C\left(\|\underset{\sim}{\underset{\sim}{v}}\|_{k}+\left\|q_{1}\right\|_{L^{2}(\Omega)}\right)\left(\left\|{\underset{\sim}{2}}_{2}\right\|_{k}+\left\|q_{2}\right\|_{L^{2}(\Omega)}\right),
\end{aligned}
$$

where the nonconforming energy norm $\|\cdot\|_{k}$ on $\underset{\sim}{H}{ }_{\perp}^{1}(\Omega)+\underset{\sim}{\underset{k}{\perp}}$ is defined by

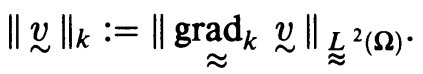

Henceforth, $C$ (with or without subscripts) denotes a generic positive constant independent of the Lamé constants and the mesh parameter $k$.

We shall show in $\S 2$ that $(1.26)$ is uniquely solvable and derive the following discretization error estimate:

$$
\begin{aligned}
& \left\|\underset{\sim}{\mathcal{u}}-{\underset{\sim}{u}}_{k}\right\|_{\underline{\sim}^{2}(\Omega)}+h_{k}\left(\left\|\underset{\sim}{\mathcal{u}}-{\underset{\sim}{u}}_{k}\right\|_{k}+\left\|p-p_{k}\right\|_{L^{2}(\Omega)}\right) \\
& \leq C h_{k}^{2}\left\{\|\underset{\sim}{f}\|_{\mathcal{\sim}^{2}(\Omega)}+\sum_{i=1}^{n}\|\underset{\sim}{g}\|_{\sim^{1 / 2}\left(\Gamma_{i}\right)}\right\} .
\end{aligned}
$$

In this paper we will develop an optimal-order multigrid method for solving (1.26). Let $n_{k}$ be the dimension of $\underset{\sim}{V} \stackrel{\perp}{k} \times Q_{k-1}$. Our full multigrid algorithm will yield an approximate solution $\left(\underset{\sim}{\underset{u}{*}}{ }_{k}^{*} p_{k}^{*}\right)$ to $(1.26)$ in $\mathscr{O}\left(n_{k}\right)$ steps such that

$$
\begin{aligned}
& \left\|{\underset{\sim}{u}}_{k}-{\underset{\sim}{u_{k}}}_{k}^{*}\right\|_{\mathcal{L}^{2}(\Omega)}+h_{k}\left(\left\|u_{\sim_{k}}-{\underset{\sim}{u_{k}}}_{k}^{*}\right\|_{k}+\left\|p_{k}-p_{k}^{*}\right\|_{L^{2}(\Omega)}\right) \\
& \leq C h_{k}^{2}\left\{\|\underset{\sim}{f}\|_{\mathcal{\sim}^{2}(\Omega)}+\sum_{i=1}^{n}\|\underset{\sim}{g}\|_{\sim^{1 / 2}\left(\Gamma_{i}\right)}\right\} \text {. }
\end{aligned}
$$

Since the constant $C$ in (1.32) does not depend on the Lamé constants, the performance of our multigrid method will not deteriorate as the material becomes nearly incompressible. As documented in [11], for nearly incompressible linear elasticity problems, the standard multigrid method using conforming bilinear finite elements requires an extremely large number of smoothing steps in order to achieve convergence. Our algorithm converges with a small number of smoothing steps, independent of the Poisson ratio.

The rest of this paper is organized as follows. We establish the discretization error estimate (1.31) in $\S 2$. Since the finite element space $\underset{\sim}{V}$ is nonconforming, appropriate intergrid transfer operators must be chosen. This is done in $\S 3$, where the mesh-dependent norms are also defined. The estimates for the intergrid transfer operators established in this section are crucial to the convergence analysis of the multigrid method. In $\S 4$ we define the multigrid algorithm. The convergence results are stated in $\S 5$. The details of the proofs are found in the supplement to this paper. Results of some numerical experiments are reported in $\S 6$.

\section{THE MIXED METHOD}

The first ingredient in establishing directly the unique solvability of (1.22) is the following well-known Korn's second inequality (cf. [10]): 
There exists a positive constant $C$ such that

$$
\|\underset{\approx}{\epsilon}(\underset{\sim}{v})\|_{\underset{\sim}{{ }^{2}(\Omega)}} \geq C|\underset{\sim}{v}|_{\underset{\sim}{H}{ }^{1}(\Omega)} \quad \forall \underset{\sim}{v} \in \underset{\sim}{\hat{\sim}^{1}}(\Omega) .
$$

Using (1.11) and (1.14), we deduce that there exists a positive constant $C$ such that

$$
\|\underset{\approx}{\epsilon}(\underset{\sim}{v})\|_{\underset{\sim}{L^{2}(\Omega)}} \geq C|\underset{\sim}{v}|_{\underset{\sim}{H}(\Omega)} \quad \forall \underset{\sim}{v} \in \underset{\sim}{H}{ }_{\perp}^{1}(\Omega)
$$

The second ingredient is the following property of the divergence operator.

Lemma 2.1. Given any $q \in L^{2}(\Omega)$, there exists $\underset{\sim}{v} \in \underset{\sim}{H}{ }_{\perp}^{1}$ such that

$$
\operatorname{div} \underset{\sim}{v}=q
$$

and

$$
\|\underset{\sim}{v}\|_{\sim^{1}(\Omega)} \leq C_{\Omega}\|q\|_{L^{2}(\Omega)}
$$

Proof. Let $D$ be an open disc that contains $\bar{\Omega}$. Extend $q$ to be zero on $D \backslash \Omega$. Let $\zeta \in H^{2}(\Omega)$ be the solution of

$$
\Delta \zeta=q \text { in } D, \quad \zeta=0 \text { on } \partial D .
$$

Then from elliptic regularity (cf. [9, Theorem 4.2.1]) we have

$$
\|\zeta\|_{H^{2}(D)} \leq C_{\Omega}\|q\|_{L^{2}(D)}
$$

Let (cf. (1.6))

$$
\underset{\sim}{v}=\left.\underset{\sim}{\operatorname{grad}} \zeta\right|_{\Omega}-\sum_{i=1}^{3}\left(\int_{\Omega} \underset{\sim}{\operatorname{grad}} \zeta \cdot \underset{\sim}{\psi} d x\right) \underset{\sim}{\psi_{i}} .
$$

It is clear that $\underset{\sim}{v} \in{\underset{\sim}{H}}_{\perp}^{1}(\Omega)$. The lemma now follows from (2.5) and (2.6).

Proposition 2.2. There exists a positive constant $C$, which depends only on $\Omega$, such that for any $\left({\underset{\sim}{v}}_{1}, q_{1}\right) \in \underset{\sim}{H} \underset{\perp}{1} \times L^{2}(\Omega)$,

$$
\begin{aligned}
& \sup _{\left(\underset{\sim}{v}, q_{2}\right) \in{\underset{\sim}{H_{\perp}}}_{\perp} \times L^{2}(\Omega) \backslash\{(\underset{\sim}{(0}, 0)\}} \frac{\left|\mathscr{B}\left(\left({\underset{\sim}{v}}_{1}, q_{1}\right),\left({\underset{\sim}{v}}_{2}, q_{2}\right)\right)\right|}{|\underset{\sim}{\underset{\sim}{v}}|_{H^{1}(\Omega)}+\left\|q_{2}\right\|_{L^{2}(\Omega)}} \\
& \geq C\left(|\underset{\sim 1}{\underset{\sim}{v}}|_{{\underset{\sim}{1}}^{1}(\Omega)}+\left\|q_{1}\right\|_{L^{2}(\Omega)}\right) .
\end{aligned}
$$

Proof. Given $\left({\underset{\sim}{v}}_{1}, q_{1}\right) \in{\underset{\sim}{H}}_{\perp}^{1} \times L^{2}(\Omega)$, let

$$
s=\sup _{\left({\underset{\sim}{v}}_{2}, q_{2}\right) \in{\underset{\sim}{H}}_{\perp}{ }_{\perp} \times L^{2}(\Omega) \backslash\{(\underset{\sim}{0}, 0)\}} \frac{\left|\mathscr{B}\left(\left({\underset{\sim}{v}}_{1}, q_{1}\right),\left({\underset{\sim}{v}}_{2}, q_{2}\right)\right)\right|}{\left|{\underset{\sim}{v}}_{2}\right|{\underset{\sim}{H}}^{1}(\Omega)+\left\|q_{2}\right\|_{L^{2}(\Omega)}} .
$$

First we consider the case where $q_{1}=\gamma \operatorname{div} \underset{\sim_{1}}{v}$. By Korn's second inequality,

$$
\begin{aligned}
\mathscr{B}\left(\left(\underset{\sim 1}{v}, q_{1}\right),(\underset{\sim 1}{v}, 0)\right) & =\int_{\Omega}\left\{\underset{\sim}{\epsilon}(\underset{\sim 1}{v}): \underset{\approx}{\epsilon}(\underset{\sim 1}{v})+\gamma(\operatorname{div} \underset{\sim 1}{v})^{2}\right\} d x \\
& \geq \int_{\Omega} \underset{\approx}{\epsilon}(\underset{\sim 1}{v}): \underset{\approx}{\epsilon}(\underset{\sim 1}{v}) d x \geq C|\underset{\sim 1}{v}|_{\sim^{1}(\Omega)}^{2} .
\end{aligned}
$$


Therefore,

$$
s \geq C|\underset{\sim}{v}|_{\underset{\sim}{H}(\Omega)}
$$

By Lemma 2.1, there exists $\underset{\sim}{v} \in \underset{\sim}{H}{ }_{\perp}^{1}$ such that

$$
\operatorname{div} \underset{\sim}{v}=q_{1} \text { and }\|\underset{\sim}{v}\|_{\sim^{1}(\Omega)} \leq C\left\|q_{1}\right\|_{L^{2}(\Omega)}
$$

Using the definition of $s,(2.9)$, and (2.10), we have

$$
\begin{aligned}
\left\|q_{1}\right\|_{L^{2}(\Omega)}^{2} & \leq\left|\mathscr{B}\left(\left(\underset{\sim}{v}, q_{1}\right),(\underset{\sim}{v}, 0)\right)\right|+\left|\int_{\Omega} \underset{\approx}{\epsilon}(\underset{\sim}{v}): \underset{\approx}{\epsilon}(\underset{\sim}{v}) d x\right| \\
& \leq\left. s\left|\underset{\sim}{\left.\right|_{H^{1}}(\Omega)}+\right| \underset{\sim}{v}\right|_{H^{1}(\Omega)}|\underset{\sim}{v}|_{H^{1}(\Omega)} \leq C s\left\|q_{1}\right\|_{L^{2}(\Omega)} .
\end{aligned}
$$

Therefore,

$$
\left\|q_{1}\right\|_{L^{2}(\Omega)} \leq C s .
$$

Combining (2.9) and (2.11), we obtain (2.8) in the special case where $q_{1}=$ $\gamma \operatorname{div} \underset{\sim}{v}$.

We now turn to the general case. Given $\left({\underset{\sim}{v}}_{1}, q_{1}\right) \in{\underset{\sim}{H}}_{\perp}^{1} \times L^{2}(\Omega)$, by Lemma 2.1 there exists $\underset{\sim}{w} \in \underset{\sim}{H}{ }_{\perp}^{1}$ such that

(2.12) $\operatorname{div} \underset{\sim}{w}=\frac{1}{\gamma} q_{1}-\operatorname{div} \underset{\sim}{v}{ }_{1}$ and $\|\underset{\sim}{w}\|_{\sim^{\prime}{ }^{\prime}(\Omega)} \leq C\left\|\frac{1}{\gamma} q_{1}-\operatorname{div} \underset{\sim}{v}\right\|_{L^{2}(\Omega)}$.

Then $\gamma \operatorname{div}(\underset{\sim}{v}+\underset{\sim}{w})=q_{1}$. From the special case, we know that

$$
\begin{aligned}
& \sup _{\left(\underset{\sim}{v}, q_{2}\right) \in{\underset{\sim}{H_{\perp}}}_{\perp} \times L^{2}(\Omega) \backslash\{(\underset{\sim}{0}, 0)\}} \frac{\left|\mathscr{B}\left(\left({\underset{\sim}{v}}_{1}+\underset{\sim}{w}, q_{1}\right),\left(\underset{\sim}{v}, q_{2}\right)\right)\right|}{|\underset{\sim}{v}|_{\sim^{1}(\Omega)}+\left\|q_{2}\right\|_{L^{2}(\Omega)}} \\
& \geq C\left(|\underset{\sim 1}{v}+\underset{\sim}{w}|_{H^{1}(\Omega)}+\left\|q_{1}\right\|_{L^{2}(\Omega)}\right) .
\end{aligned}
$$

Therefore, by (2.13), the bilinearity of $\mathscr{B}$ and (1.23), we have

$$
\begin{aligned}
|\underset{\sim 1}{v}|_{H^{1}(\Omega)}+\left\|q_{1}\right\|_{L^{2}(\Omega)} & \leq\left[|\underset{\sim 1}{v}+\underset{\sim}{w}|_{\sim_{H^{1}(\Omega)}}+\left\|q_{1}\right\|_{L^{2}(\Omega)}\right]+|\underset{\sim}{w}|_{H^{1}(\Omega)} \\
& \leq s+C \mid \underset{\sim}{\mid \underset{H^{1}(\Omega)}{\mid}} .
\end{aligned}
$$

On the other hand, by the definition of $\mathscr{B}$,

$$
\begin{aligned}
\left\|\frac{1}{\gamma} q_{1}-\operatorname{div} \underset{\sim}{v}\right\|_{L^{2}(\Omega)}^{2} & =\mathscr{B}\left(\left({\underset{\sim}{v}}_{1}, q_{1}\right),\left(\underset{\sim}{0}, \operatorname{div} \underset{\sim}{\underset{\sim}{v}}-\frac{1}{\gamma} q_{1}\right)\right) \\
& \leq s\left\|\frac{1}{\gamma} q_{1}-\operatorname{div} \underset{\sim}{\underset{\sim}{v}}\right\|_{L^{2}(\Omega)} .
\end{aligned}
$$

Combining (2.12) and (2.15), we see that

$$
\|\underset{\sim}{w}\|_{\underset{\sim}{H}{ }^{1}(\Omega)} \leq C s .
$$

The proposition now follows from (2.14) and (2.16).

The following corollary is a consequence of Proposition 2.2 and Friedrichs' inequality. 
Corollary 2.3. Given any bounded linear functional $F$ on $\underset{\sim}{H}{ }_{\perp}^{1} \times L^{2}(\Omega)$, there is a unique $\left(\underset{\sim}{v}, q_{1}\right) \in \underset{\sim}{\underset{\sim}{H}}{ }_{\perp}^{1} \times L^{2}(\Omega)$ such that

(2.17) $\mathscr{B}\left(\left({\underset{\sim}{v}}_{1}, q_{1}\right),\left({\underset{\sim}{v}}_{2}^{v}, q_{2}\right)\right)=F\left(\left({\underset{\sim}{v}}_{2}, q_{2}\right)\right) \quad \forall\left({\underset{\sim}{v}}_{2}, q_{2}\right) \in \underset{\sim}{H_{\perp}} \times L^{2}(\Omega)$.

In particular, equation (1.22) is uniquely solvable.

Similarly, there are two ingredients in establishing the unique solvability of the discretized equation (1.26), namely a discrete Korn's second inequality and an analog of Lemma 2.1 .

We begin with the discrete Korn's second inequality. Let $\underset{\sim}{\hat{V}}=\{\underset{\sim}{v} \in \underset{\sim}{V}$ : $\int_{\Omega} \underset{\sim}{v} d x=\underset{\sim}{0}$ and $\left.\int_{\Omega} \operatorname{rot}_{k} \underset{\sim}{v} d x=0\right\}$. Analogous to (1.7) and (1.8), define

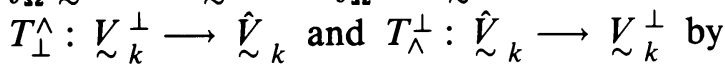

$$
T_{\perp}^{\wedge}(\underset{\sim}{v})=\underset{\sim}{v}-\frac{\omega}{2|\Omega|}\left(\int_{\Omega} \operatorname{rot}_{k} \underset{\sim}{v} d x\right) \underset{\sim}{\psi}
$$

and

$$
T_{\wedge}^{\perp}(\underset{\sim}{v})=\underset{\sim}{v}-\left(\int_{\Omega} \underset{\sim}{v} \cdot{\underset{\sim}{\psi}}_{3} d x\right){\underset{\sim}{\psi}}_{3} .
$$

Since $\underset{\sim}{\epsilon}{ }_{k}^{*}\left({\underset{\sim}{3}}_{3}\right)=\underset{\sim}{\epsilon}\left(\underset{\sim}{\psi_{3}}\right)=\underset{\approx}{0}$, we have

$$
\underset{\approx}{\epsilon}(\underset{\sim}{v})=\underset{\approx}{\epsilon}\left(T_{\perp}^{\wedge}(\underset{\sim}{v})\right) \quad \forall \underset{\sim}{v} \in \underset{\sim}{\underset{\sim}{\perp}}
$$

By the discrete Friedrichs' inequality (cf. [14]) for ${\underset{\sim}{V}}_{k}$, there exist positive constants $C_{1}$ and $C_{2}$ such that

$$
C_{1}\left\|T_{\perp}^{\wedge}(\underset{\sim}{v})\right\|_{k} \leq\|\underset{\sim}{v}\|_{k} \leq C_{2}\left\|T_{\perp}^{\wedge}(\underset{\sim}{v})\right\|_{k} \quad \forall \underset{\sim}{v} \in \underset{\sim}{\underset{\sim}{\perp}}
$$

The proof of the following discrete Korn's second inequality can be found in [6].

Lemma 2.4. There exists a positive constant $C$ such that

$$
\left\|\underset{\sim}{\epsilon}{ }_{k}^{*}(\underset{\sim}{v})\right\|_{\underset{\sim}{L^{2}(\Omega)}} \geq C\|\underset{\sim}{v}\|_{k} \quad \forall \underset{\sim}{v} \in \hat{\sim}_{k}
$$

With the aid of the operator $T_{\perp}^{\wedge}$ we can translate Lemma 2.4 into a result

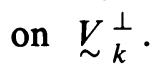

Corollary 2.5. There exists a positive constant $C$ such that

$$
\left.\| \underset{\sim}{\epsilon}{\underset{\sim}{v}}^{*}\right)\left\|_{\underset{\sim}{L^{2}(\Omega)}} \geq C\right\| \underset{\sim}{v} \|_{k} \quad \forall \underset{\sim}{v} \in \underset{\sim}{V} \underset{k}{\perp}
$$

Proof. Let $\underset{\sim}{v} \in \underset{\sim}{V} \underset{k}{\perp}$. Then, using (2.20), Lemma 2.4, and (2.21), we get

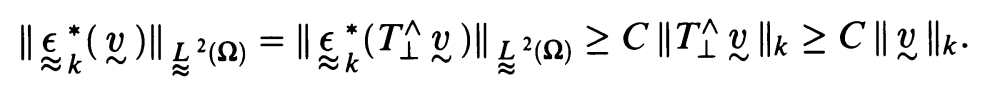

There is an interpolation operator $\Pi_{k}: \underset{\sim}{H}{ }^{1}(\Omega) \longrightarrow \underset{\sim}{V}$ defined by (cf. [5])

$$
\left[\Pi_{k}(\underset{\sim}{\phi})\right]\left(m_{e}\right):=\frac{1}{|e|} \int_{e} \underset{\sim}{\phi} d s
$$

at the midpoints $m_{e}$ of the edge $e$ in $\mathscr{T}_{k}$. 
A simple homogeneity argument shows that

$$
\left\|\Pi_{k} \underset{\sim}{\phi}\right\|_{k} \leq C|\underset{\sim}{\phi}|_{\sim^{1}(\Omega)} \forall \underset{\sim}{\phi} \in \underset{\sim}{H^{1}(\Omega) .}
$$

It follows from a simple calculation (cf. [5]) that

$$
\operatorname{div}_{k}\left[\Pi_{k} \underset{\sim}{\phi}\right]=P_{k}(\operatorname{div} \underset{\sim}{\phi}) \quad \forall \underset{\sim}{\phi} \in \underset{\sim}{H}{ }^{1}(\Omega)
$$

and

$$
\operatorname{rot}_{k}\left[\Pi_{k} \underset{\sim}{\phi}\right]=P_{k}(\operatorname{rot} \underset{\sim}{\phi}) \quad \forall \underset{\sim}{\phi} \in \underset{\sim}{H}{ }^{1}(\Omega) \text {. }
$$

The following interpolation error estimate also holds (cf. [5]):

(2.26) $\left\|\underset{\sim}{\phi}-\Pi_{k} \underset{\sim}{\phi}\right\|_{\mathcal{\sim}^{2}(\Omega)}+h_{k}\left\|\underset{\sim}{\phi}-\Pi_{k} \underset{\sim}{\phi}\right\|_{k} \leq C h_{k}^{2}|\underset{\sim}{\phi}|_{\sim}{ }^{2}(\Omega) \quad \forall \underset{\sim}{\phi} \in \underset{\sim}{H^{2}}(\Omega)$.

Let $\Pi_{k}^{\perp}$ be defined by

$$
\Pi_{k}^{\perp}(\underset{\sim}{\phi}):=\Pi_{k}(\underset{\sim}{\phi})-\sum_{i=1}^{3}\left(\int_{\Omega} \Pi_{k}(\underset{\sim}{\phi}) \cdot \underset{\sim}{\underset{\sim}{\psi}} d x\right) \underset{\sim}{\underset{\sim}{\psi}} \quad \forall \underset{\sim}{\phi} \in \underset{\sim}{H_{\perp}} .
$$

It follows that $\Pi_{k}^{\perp}$ maps $\underset{\sim}{H}{ }_{\perp}^{1}$ into $\underset{\sim}{V} \stackrel{\perp}{\perp}$. Moreover, we still have

$$
\begin{aligned}
& \left\|\Pi_{k}^{\perp} \underset{\sim}{\phi}\right\|_{k} \leq C|\underset{\sim}{\phi}|_{\sim}^{{ }^{1}(\Omega)} \quad \forall \underset{\sim}{\phi} \in \underset{\sim}{H_{\perp}}{ }^{1}, \\
& \operatorname{div}_{k}\left[\Pi_{k}^{\perp} \underset{\sim}{\phi}\right]=P_{k}(\operatorname{div} \underset{\sim}{\phi}) \quad \forall \underset{\sim}{\phi} \in \underset{\sim}{H}{ }_{\perp}^{1},
\end{aligned}
$$

and

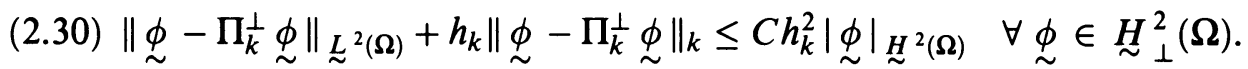

Also, a standard interpolation error estimate (cf. [4, Theorem 3.1.6]) shows that

$$
\left\|\psi-P_{k-1} \psi\right\|_{L^{2}(\Omega)} \leq C h_{k}|\psi|_{H^{1}(\Omega)} \quad \forall \psi \in H^{1}(\Omega) .
$$

The following lemma is the discrete analog of Lemma 2.1.

Lemma 2.6. There exists a positive constant $C$ such that, given any $q \in Q_{k}$, there exists $\underset{\sim}{v} \in \underset{\sim}{\underset{\sim}{\perp}}$ which satisfies

$$
\operatorname{div}_{k} \underset{\sim}{v}=q
$$

and

$$
\|\underset{\sim}{v}\|_{k} \leq C\|q\|_{L^{2}(\Omega)}
$$

Proof. From Lemma 2.1, there exists $\underset{\sim}{w} \in \underset{\sim}{H}{ }_{\perp}^{1}$ such that

$$
\operatorname{div} \underset{\sim}{w}=q
$$

and

$$
\|\underset{\sim}{w}\|_{\sim_{\sim}^{\prime}(\Omega)} \leq C\|q\|_{L^{2}(\Omega)} .
$$

Let $\underset{\sim}{v}=\Pi_{k}^{\perp} \underset{\sim}{w}$. Then by (2.29) and (2.34) we have

and by (2.28) and (2.35),

$$
\operatorname{div}_{k} \underset{\sim}{v}=q,
$$

$$
\|\underset{\sim}{v}\|_{k} \leq C\|q\|_{L^{2}(\Omega)} .
$$

With Corollary 2.5 and Lemma 2.6 in place, the following discrete version of Proposition 2.2 follows verbatim. 
Proposition 2.7. There exists a positive constant $C$ such that for any $\left(\underset{\sim}{v}, q_{1}\right) \in$ $\underset{\sim}{V} \stackrel{\perp}{\sim} \times Q_{k-1}$,

$$
\begin{aligned}
\sup _{\left(\underset{\sim}{v}, q_{2}\right) \in{\underset{\sim}{\sim}}_{k}^{\perp} \times Q_{k-1} \backslash(\{\underset{\sim}{0}, 0)\}} \frac{\left|\mathscr{B}_{k}\left(\left({\underset{\sim}{v}}_{1}, q_{1}\right),\left({\underset{\sim}{v}}_{2}, q_{2}\right)\right)\right|}{\|\underset{\sim}{v}\|_{k}+\left\|q_{2}\right\|_{L^{2}(\Omega)}} \\
\geq C\left(\left\|{\underset{\sim}{v}}_{1}\right\|_{k}+\left\|q_{1}\right\|_{L^{2}(\Omega)}\right) .
\end{aligned}
$$

Corollary 2.8. Given any linear functional $F$ on $\underset{\sim}{V} \underset{k}{\perp} \times Q_{k-1}$, there exists a unique $\left(\underset{\sim}{v}, q_{1}\right) \in \underset{\sim}{V} \underset{k}{\perp} \times Q_{k-1}$ such that

(2.37) $\mathscr{B}\left(\left({\underset{\sim}{v}}_{1}, q_{1}\right),\left({\underset{\sim}{v}}_{2}, q_{2}\right)\right)=F\left(\left({\underset{\sim}{v}}_{2}, q_{2}\right)\right) \quad \forall\left({\underset{\sim}{v}}_{2}, q_{2}\right) \in \underset{\sim}{V} \stackrel{\perp}{k} \times Q_{k-1}$

In particular, the discretized equation (1.26) is uniquely solvable.

We can now establish the discretization error estimate.

Theorem 2.9. If $(\underset{\sim}{u}, p) \in \underset{\sim}{H}{ }_{\perp}^{1} \times L^{2}(\Omega)$ solves (1.22) and $\left({\underset{\sim}{u}}_{k}, p_{k}\right) \in \underset{\sim}{\stackrel{\perp}{\perp} \times}$ $Q_{k-1}$ solves $(1.26)$, then

(2.38) $\quad\left\|\underset{\sim}{u}-\underset{\sim}{u_{k}}\right\|_{k}+\left\|p-p_{k}\right\|_{L^{2}(\Omega)} \leq C h_{k}\left(\|\underset{\sim}{f}\|_{\sim^{2}(\Omega)}+\sum_{i=1}^{n}\|\underset{\sim}{g}\|_{\sim_{\sim}^{1 / 2}\left(\Gamma_{i}\right)}\right)$.

Proof. We follow the ideas of Scott (cf. [12] and [13, pp. 178-179]) for estimating the errors caused by variational crimes. Given any $(\underset{\sim}{v}, q) \in \underset{\sim}{V} \underset{k}{\perp} \times Q_{k-1}$, using (2.36), we have

$$
\begin{aligned}
& \left\|\underset{\sim}{u}-{\underset{\sim}{u}}_{k}\right\|_{k}+\left\|p-p_{k}\right\|_{L^{2}(\Omega)} \\
& \leq\|\underset{\sim}{u}-\underset{\sim}{v}\|_{k}+\|p-q\|_{L^{2}(\Omega)}+\left\|\underset{\sim}{v}-{\underset{\sim}{u}}_{k}\right\|_{k}+\left\|q-p_{k}\right\|_{L^{2}(\Omega)} \\
& \leq\|\underset{\sim}{u}-\underset{\sim}{v}\|_{k}+\|p-q\|_{L^{2}(\Omega)} \\
& +C \sup _{(\underset{\sim}{w}, r) \in V_{\sim}^{\perp} \times Q_{k-1} \backslash\{(\underset{\sim}{0}, 0)\}} \frac{\left|\mathscr{B}_{k}\left(\left(\underset{\sim}{v}-{\underset{\sim}{u}}_{k}, q-p_{k}\right),(\underset{\sim}{w}, r)\right)\right|}{\|\underset{\sim}{w}\|_{k}+\|r\|_{L^{2}(\Omega)}} .
\end{aligned}
$$

By (1.29) and (1.26) we have

$$
\begin{aligned}
& \frac{\left|\mathscr{B}_{k}\left(\left(\underset{\sim}{v}-\underset{\sim}{u}, q-p_{k}\right),(\underset{\sim}{w}, r)\right)\right|}{\|\underset{\sim}{w}\|_{k}+\|r\|_{L^{2}(\Omega)}} \\
& \leq C\left(\underset{\sim}{u}-\underset{\sim}{v}\left\|_{k}+\right\| p-q \|_{L^{2}(\Omega)}\right) \\
& \quad+C \frac{\left|\mathscr{B}_{k}((\underset{\sim}{u}, p),(\underset{\sim}{w}, r))-\frac{1}{2 \mu}\left[\int_{\Omega} \underset{\sim}{f} \cdot \underset{\sim}{w} d x+\left.\sum_{i=1}^{n} \int_{\Gamma_{i}} \underset{\sim}{g} \cdot \underset{\sim}{w}\right|_{\Gamma_{i}} d s\right]\right|}{\|\underset{\sim}{w}\|_{k}+\|r\|_{L^{2}(\Omega)}}
\end{aligned}
$$


Combining (2.39) and (2.40), we have

(2.41)

$$
\begin{aligned}
\left\|\underset{\sim}{\mathcal{u}}-{\underset{\sim}{u}}_{k}\right\|_{k}+\left\|p-p_{k}\right\|_{L^{2}(\Omega)} & \\
\leq & C\left(\|\underset{\sim}{\mathcal{u}}-\underset{\sim}{v}\|_{k}+\|p-q\|_{L^{2}(\Omega)}\right) \\
& +C \sup \frac{\left|\mathscr{B}_{k}((\underset{\sim}{u}, p),(\underset{\sim}{w}, r))-\frac{1}{2 \mu}\left[\int_{\Omega} \underset{\sim}{f} \cdot \underset{\sim}{w} d x+\left.\sum_{i=1}^{n} \int_{\Gamma_{i}}^{\underset{\sim}{g}} \cdot \underset{\sim}{w}\right|_{\Gamma_{i}} d s\right]\right|}{\|\underset{\sim}{w}\|_{k}+\|r\|_{L^{2}(\Omega)}},
\end{aligned}
$$

where the supremum term is taken over all $(\underset{\sim}{w}, r) \in \underset{\sim}{V} \underset{k}{\perp} \times Q_{k-1} \backslash\{(\underset{\sim}{0}, 0)\}$. The supremum term on the right-hand side of (2.41) measures the effect of the nonconformity of $\underset{\sim}{V} \stackrel{\perp}{ }$ and the effect of reduced integration built into the definition of $\underset{\approx}{\underset{\sim}{\epsilon}}\left(\mathrm{cf}\right.$. (1.28)). It is bounded by $C h_{k} \mid \underset{\sim}{\left.\underset{\sim}{u}\right|_{\underset{\sim}{2}(\Omega)}}$ (cf. [3, 5]). If we take $(\underset{\sim}{v}, q)$ to be $\left(\Pi_{k}^{\perp} \underset{\sim}{u}, P_{k-1} p\right)$, then $(2.30),(2.31)$ and (2.41) imply that

$$
\begin{aligned}
\| \underset{\sim}{u}- & -\underset{\sim}{u}\left\|_{k}+\right\| p-p_{k} \|_{L^{2}(\Omega)} \\
& \leq C\left(\left\|\underset{\sim}{u}-\Pi_{k}^{\perp} \underset{\sim}{\sim}\right\|_{k}+\left\|p-P_{k-1} p\right\|_{L^{2}(\Omega)}+h_{k} \mid \underset{\sim}{\mid \underset{H^{2}(\Omega)}{u}}\right) \\
& \leq C h_{k}\left(|\underline{\sim}|_{\mathcal{H}^{2}(\Omega)}+|p|_{H^{1}(\Omega)}\right) .
\end{aligned}
$$

Since $p=\gamma \operatorname{div} \underset{\sim}{\sim}$, the theorem follows from (2.42) and the elliptic regularity estimate (1.20).

Theorem 2.10. There exists a positive constant $C$ such that

$$
\|\underset{\sim}{u}-\underset{\sim}{u}\|_{k} \|_{L^{2}(\Omega)} \leq C h_{k}^{2}\left(\|\underset{\sim}{f}\|_{L^{2}(\Omega)}+\sum_{i=1}^{n}\|\underset{\sim}{g}\|_{i}{ }_{\sim}^{1 / 2\left(\Gamma_{i}\right)}\right) .
$$

Proof. We use a duality argument. Since $\underset{\sim}{u}-{\underset{\sim}{u}}_{k} \in \underset{\sim}{L_{\perp}^{2}}=\left\{\underset{\sim}{v} \in \underset{\sim}{L^{2}}(\Omega)\right.$ : $\left.\int_{\Omega} \underset{\sim}{v} \cdot \underset{\sim}{w} d x=0 \quad \forall \underset{\sim}{w} \in \mathbf{R M}\right\}$, we can write

$$
\left\|\underset{\sim}{u}-{\underset{\sim}{u}}_{k}\right\|_{\mathcal{\sim}^{2}(\Omega)}=\sup _{\underset{\sim}{w} \in{\underset{\sim}{L}}_{\perp}^{2}(\Omega) \backslash\{\underset{\sim}{0}\}} \frac{\left|\int_{\Omega}(\underset{\sim}{u}-\underset{\sim}{u}) \cdot \underset{\sim}{w} d x\right|}{\|\underset{\sim}{w}\|_{L^{2}(\Omega)}} .
$$

Let $\underset{\sim}{w} \in{\underset{\sim}{L}}_{\perp}^{2}(\Omega)$. Then

$$
-\operatorname{div}\{2 \mu \underset{\sim}{\epsilon}(\zeta)+\lambda \operatorname{tr} \underset{\sim}{\epsilon}(\underset{\sim}{\zeta})) \underset{\approx}{\delta}\}=\underset{\sim}{w} \text { in } \Omega,
$$

$$
(2 \mu \underset{\sim}{\epsilon}(\zeta)+\lambda \operatorname{tr} \underset{\sim}{(\underset{\sim}{(\zeta)})}) \underset{\sim}{\delta}) \underset{\sim}{\nu_{i}} \mid \Gamma_{i}=\underset{\sim}{0}, \quad 1 \leq i \leq n,
$$

has a unique solution $\underset{\sim}{\zeta} \in{\underset{\sim}{H}}_{\perp}^{2}(\Omega)$ because conditions (1.16) and (1.17) are satisfied for $(\underset{\sim}{w}, 0)$.

The boundary value problem $(2.45)$ is equivalent to

$$
\mathscr{B}((\underset{\sim}{\zeta}, \xi),(\underset{\sim}{v}, q))=\frac{1}{2 \mu} \int_{\Omega} \underset{\sim}{w} \cdot \underset{\sim}{v} d x
$$

for all $(\underset{\sim}{v}, q) \in \underset{\sim}{H}{ }_{\perp}^{1} \times L^{2}(\Omega)$, where $\xi=(\lambda /(2 \mu)) \operatorname{div} \underset{\sim}{\zeta}$. The elliptic regularity estimate (1.20) implies that

$$
\|\underset{\sim}{\zeta}\|_{H^{2}(\Omega)}+\|\xi\|_{H^{1}(\Omega)} \leq C\|\underset{\sim}{w}\|_{\sim^{2}(\Omega)} .
$$


Let $\left(\underset{\sim}{\zeta}, \xi_{k}\right) \in \underset{\sim}{V} \underset{k}{\perp} \times Q_{k-1}$ satisfy

(2.48) $\mathscr{B}_{k}\left(\left(\underset{\sim}{\zeta}, \xi_{k}\right),(\underset{\sim}{v}, q)\right)=\frac{1}{2 \mu} \int_{\Omega} \underset{\sim}{w} \cdot \underset{\sim}{v} d x \quad \forall(\underset{\sim}{v}, q) \in \underset{\sim}{V} \stackrel{\perp}{k} \times Q_{k-1}$

Theorem 2.9 implies that

$$
\|\underset{\sim}{\zeta}-\underset{\sim}{\zeta}\|_{k}+\left\|\xi-\xi_{k}\right\|_{L^{2}(\Omega)} \leq C h_{k}\|\underset{\sim}{w}\|_{L^{2}(\Omega)}
$$

The interpolation error estimates (2.30)-(2.31) and (2.47) imply that

$$
\begin{aligned}
& \left\|\underset{\sim}{\zeta}-\Pi_{k}^{\perp} \underset{\sim}{\zeta}\right\|_{\mathcal{L}^{2}(\Omega)}+h_{k}\left\|\underset{\sim}{\zeta}-\Pi_{k}^{\perp} \underset{\sim}{\zeta}\right\|_{k}+h_{k}\left\|\xi-P_{k-1} \xi\right\|_{L^{2}(\Omega)} \\
& \leq C h_{k}^{2}\|\underset{\sim}{w}\|_{\mathcal{L}^{2}(\Omega)} .
\end{aligned}
$$

Using (1.29), (2.46) and (2.48), we have

$$
\begin{aligned}
& \left|\frac{1}{2 \mu} \int_{\Omega}\left(\underset{\sim}{\sim}-{\underset{\sim}{u}}_{k}\right) \cdot \underset{\sim}{w} d x\right| \\
& \left.\left.=\mid \mathscr{B}_{k}(\underset{\sim}{(\underset{\sim}{\zeta}}, \boldsymbol{\xi}),(\underset{\sim}{u}, p)\right)-\mathscr{B}_{k}\left(\underset{\sim_{k}}{(\zeta}, \xi_{k}\right),\left({\underset{\sim}{k}}_{k}, p_{k}\right)\right) \\
& +\mathscr{B}((\underset{\sim}{\zeta}, \xi),(\underset{\sim}{u}, p))-\mathscr{B}_{k}((\underset{\sim}{\zeta}, \xi),(\underset{\sim}{u}, p)) \mid \\
& =\mid \mathscr{B}_{k}\left(\left(\underset{\sim}{\zeta}-{\underset{\sim}{k}}_{k}, \xi-\xi_{k}\right),\left(\underset{\sim}{u}-\Pi_{k}^{\perp} \underset{\sim}{\mathcal{u}}, p-P_{k-1} p\right)\right) \\
& +\mathscr{B}_{k}\left(\left(\sim_{\sim}^{\zeta}-\zeta_{k}, \xi-\xi_{k}\right),\left(\Pi_{k}^{\perp} \underset{\sim}{u}, P_{k-1} p\right)\right) \\
& \left.+\mathscr{B}_{k}\left({\underset{\sim}{\zeta}}_{k}-\Pi_{k}^{\perp} \underset{\sim}{\zeta}, \xi_{k}-P_{k-1} \xi\right),\left(\underset{\sim}{u}-{\underset{\sim}{u}}_{k}, p-p_{k}\right)\right) \\
& +\mathscr{B}_{k}\left(\left(\Pi_{k}^{\perp} \underset{\sim}{\zeta}, P_{k-1} \xi\right),\left(\underset{\sim}{u}-{\underset{\sim}{u}}_{k}, p-p_{k}\right)\right) \\
& +\mathscr{B}((\underset{\sim}{\zeta}, \xi),(\underset{\sim}{u}, p))-\mathscr{B}_{k}((\underset{\sim}{\zeta}, \xi),(\underset{\sim}{u}, p)) \mid \\
& \leq C\left\{\left(\left\|\underset{\sim}{\zeta}-{\underset{\sim}{k}}_{k}\right\|_{k}+\left\|\xi-\xi_{k}\right\|_{L^{2}(\Omega)}\right)\left(\left\|\underset{\sim}{u}-\Pi_{k}^{\perp} \underset{\sim}{u}\right\|_{k}+\left\|p-P_{k-1} p\right\|_{L^{2}(\Omega)}\right)\right. \\
& \left.+\left(\left\|{\underset{\sim}{k}}_{k}-\Pi_{k}^{\perp} \underset{\sim}{\zeta}\right\|_{k}+\left\|\xi_{k}-P_{k-1} \xi\right\|_{L^{2}(\Omega)}\right)\left(\left\|\underset{\sim}{u}-{\underset{\sim}{u} k}_{k}\right\|_{k}+\left\|p-p_{k}\right\|_{L^{2}(\Omega)}\right)\right\} \\
& +\left|\mathscr{B}_{k}\left(\left(\underset{\sim}{\zeta}-\zeta_{k}, \xi-\xi_{k}\right),\left(\Pi_{k}^{\perp} \underset{\sim}{u}, P_{k-1} p\right)\right)\right| \\
& +\left|\mathscr{B}_{k}\left(\left(\Pi_{k}^{\perp} \underset{\sim}{\zeta}, P_{k-1} \xi\right),\left(\underset{\sim}{u}-{\underset{\sim}{u}}_{k}, p-p_{k}\right)\right)\right| \\
& +\left|\mathscr{B}((\underset{\sim}{\zeta}, \xi),(\underset{\sim}{u}, p))-\mathscr{B}_{k}((\underset{\sim}{\zeta}, \xi),(\underset{\sim}{u}, p))\right| .
\end{aligned}
$$

From (2.30)-(2.31), (1.20), Theorem 2.9 and (2.49)-(2.50), we know that (2.52)

$$
\begin{aligned}
& \left(\left\|\underset{\sim}{\zeta}-{\underset{\sim}{k}}_{k}\right\|_{k}+\left\|\xi-\xi_{k}\right\|_{L^{2}(\Omega)}\right)\left(\left\|\underset{\sim}{u}-\Pi_{k}^{\perp} \underset{\sim}{\perp}\right\|_{k}+\left\|p-P_{k-1} p\right\|_{L^{2}(\Omega)}\right) \\
& +\left(\left\|\sim_{k}-\Pi_{k}^{\perp} \underset{\sim}{\zeta}\right\|_{k}+\left\|\xi_{k}-P_{k-1} \xi\right\|_{L^{2}(\Omega)}\right)\left(\left\|\underset{\sim}{u}-{\underset{\sim}{u}}_{k}\right\|_{k}+\left\|p-p_{k}\right\|_{L^{2}(\Omega)}\right) \\
& \leq C h_{k}^{2}\|\underset{\sim}{w}\|_{\mathcal{L}^{2}(\Omega)}\left(\|f\|_{\mathcal{L}^{2}(\Omega)}+\sum_{i=1}^{n}\|\underset{\sim}{g}\|_{H^{1 / 2}\left(\Gamma_{i}\right)}\right) \text {. }
\end{aligned}
$$


It therefore remains to estimate

$$
\begin{aligned}
& \left|\mathscr{B}_{k}\left(\left(\underset{\sim}{\zeta}-\underset{\sim}{\zeta}, \xi \xi-\xi_{k}\right),\left(\Pi_{k}^{\perp} \underset{\sim}{\mathcal{u}}, P_{k-1} p\right)\right)\right|, \\
& \left|\mathscr{B}_{k}\left(\left(\Pi_{k}^{\perp} \underset{\sim}{\zeta}, P_{k-1} \xi\right),\left(\underset{\sim}{\mathcal{u}}-{\underset{\sim}{u}}_{k}, p-p_{k}\right)\right)\right|
\end{aligned}
$$

and

$$
\left|\mathscr{B}((\underset{\sim}{\zeta}, \xi),(\underset{\sim}{u}, p))-\mathscr{B}_{k}((\underset{\sim}{\zeta}, \xi),(\underset{\sim}{u}, p))\right| .
$$

In order to obtain a bound for $\left|\mathscr{B}_{k}\left(\left(\underset{\sim}{\zeta}-\underset{\sim}{\zeta}, \xi-\xi_{k}\right),\left(\Pi_{k}^{\perp} \underset{\sim}{u}, P_{k-1} p\right)\right)\right|$, first note that by (1.27), the fact that $\xi=\gamma \operatorname{div} \underset{\sim}{\zeta},(2.45)$ and $(2.48)$, we have (2.53)

$$
\begin{aligned}
& \mathscr{B}_{k}\left(\left(\underset{\sim}{\zeta}-\underset{\sim}{\zeta}, \xi-\xi_{k}\right),\left(\Pi_{k}^{\perp} \underset{\sim}{\mathcal{u}}, P_{k-1} p\right)\right) \\
& =\mathscr{B}_{k}\left((\underset{\sim}{\zeta}, \xi),\left(\Pi_{k}^{\perp} \underset{\sim}{u}, P_{k-1} p\right)\right)-\frac{1}{2 \mu} \int_{\Omega} \underset{\sim}{w} \cdot\left(\Pi_{k}^{\perp} \underset{\sim}{u}\right) d x \\
& =\int_{\Omega}\left[\underset{\approx k}{\underset{\sim}{\epsilon}}(\underset{\sim}{\zeta}): \underset{\approx k}{\epsilon_{*}^{*}}\left(\Pi_{k}^{\perp} \underset{\sim}{u}\right)+\xi \operatorname{div}_{k}\left(\Pi_{k}^{\perp} \underset{\sim}{u}\right)\right] d x-\frac{1}{2 \mu} \int_{\Omega} \underset{\sim}{w} \cdot\left(\Pi_{k}^{\perp} \underset{\sim}{u}\right) d x \\
& =\int_{\Omega}\left[\underset{\approx k}{\epsilon}(\underset{\sim}{\zeta}): \underset{\approx k}{\epsilon_{*}^{*}}\left(\Pi_{k}^{\perp} \underset{\sim}{u}\right)-\underset{\approx k}{\epsilon}(\underset{\sim}{\zeta}): \underset{\approx k}{\epsilon}\left(\Pi_{k}^{\perp} \underset{\sim}{u}\right)\right] d x \\
& +\int_{\Omega}\left[\underset{\approx k}{\epsilon}(\underset{\sim}{\zeta}): \underset{\approx k}{\epsilon}\left(\Pi_{k}^{\perp} \underset{\sim}{u}\right)+\operatorname{div}(\underset{\approx k}{\epsilon}(\underset{\sim}{\zeta})) \cdot \Pi_{k}^{\perp} \underset{\sim}{u}\right] d x \\
& +\int_{\Omega}\left[\frac{\lambda}{2 \mu} \operatorname{tr}(\underset{\approx}{\epsilon}(\underset{\sim}{\zeta})) \underset{\approx}{\delta}: \operatorname{grad}_{\approx}\left(\Pi_{k}^{\perp} \underset{\sim}{u}\right)+\operatorname{div}\left(\frac{\lambda}{2 \mu} \operatorname{tr}(\underset{\approx}{\epsilon}(\underset{\sim}{\zeta})) \underset{\approx}{\delta}\right) \cdot\left(\Pi_{k}^{\perp} \underset{\sim}{u}\right)\right] d x,
\end{aligned}
$$

where $\underset{\approx}{\epsilon}(\underset{\sim}{\phi}):=\frac{1}{2}\left(\operatorname{grad}_{k} \underset{\sim}{\phi}+\left(\operatorname{grad}_{k} \underset{\sim}{\phi}\right)^{t}\right)=\operatorname{grad}_{k} \underset{\sim}{\phi}-\frac{1}{2}\left(\operatorname{rot}_{k} \underset{\sim}{\phi}\right) \underset{\approx}{\chi}$. Standard nonconforming estimates (cf. [5]) $\underset{\text { and elliptic regularity }(1.20) \text { and }}{\approx} \underset{\approx}{\approx}(2.47)$ give estimates on the last two integrals, i.e.,

$$
\begin{aligned}
& \left|\int_{\Omega}\left[\underset{\approx k}{\epsilon}(\underset{\sim}{\zeta}): \underset{\approx k}{\epsilon}\left(\Pi_{k}^{\perp} \underset{\sim}{u}\right)+\operatorname{div}(\underset{\approx k}{\epsilon}(\underset{\sim}{\zeta})) \cdot \Pi_{k}^{\perp} \underset{\sim}{u}\right] d x\right| \\
& \leq\left. C h_{k}^{2} \underset{\sim}{\mid}\right|_{H^{2}(\Omega)} \mid \underset{\sim}{\mid \underset{H^{2}(\Omega)}{\mid}} \\
& \leq C h_{k}^{2}\|\underset{\sim}{w}\|_{\mathcal{L}^{2}(\Omega)}\left(\|\underset{\sim}{f}\|_{L^{2}(\Omega)}+\sum_{i=1}^{n}\|\underset{\sim}{g}\|_{\mathcal{H}^{1 / 2}\left(\Gamma_{i}\right)}\right)
\end{aligned}
$$

and

$$
\begin{aligned}
& \left|\int_{\Omega}\left[\frac{\lambda}{2 \mu} \operatorname{tr}(\underset{\approx}{\epsilon}(\underset{\sim}{\zeta})) \underset{\approx}{\delta}: \operatorname{grad}_{\approx}\left(\Pi_{k}^{\perp} \underset{\sim}{u}\right)+\underset{\sim}{\operatorname{div}}\left(\frac{\lambda}{2 \mu} \operatorname{tr}(\underset{\approx}{\epsilon}(\underset{\sim}{\zeta})) \underset{\approx}{\delta}\right) \cdot \Pi_{k}^{\perp} \underset{\sim}{u}\right] d x\right|
\end{aligned}
$$

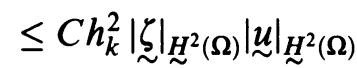

$$
\begin{aligned}
& \leq C h_{k}^{2}\|\underset{\sim}{w}\|_{\mathcal{L}^{2}(\Omega)}\left(\|\underset{\sim}{f}\|_{\underset{\sim}{L^{2}(\Omega)}}+\sum_{i=1}^{n}\|\underset{\sim}{g}\|_{\mathcal{\sim}^{1 / 2}\left(\Gamma_{i}\right)}\right) \text {. }
\end{aligned}
$$


We must still show that

$$
\begin{aligned}
& \left|\int_{\Omega}\left[\underset{\approx k}{\epsilon_{\approx}^{*}}(\underset{\sim}{\zeta}): \underset{\approx k}{\epsilon^{*}}\left(\Pi_{k}^{\perp} \underset{\sim}{u}\right)-\underset{\approx k}{\epsilon}(\underset{\sim}{\zeta}): \underset{\approx k}{\epsilon}\left(\Pi_{k}^{\perp} \underset{\sim}{u}\right)\right] d x\right| \\
& \leq C h_{k}^{2}\|\underset{\sim}{w}\|_{\mathcal{L}^{2}(\Omega)}\left(\|\underset{\sim}{f}\|_{L^{2}(\Omega)}+\sum_{i=1}^{n}\|\underset{\sim}{g}\|_{\sim_{\sim}^{1 / 2}\left(\Gamma_{i}\right)}\right) .
\end{aligned}
$$

The left-hand side of $(2.56)$ is bounded by

$$
\begin{aligned}
& \left|\int_{\Omega} \operatorname{grad}_{\approx} \zeta:\left[-\frac{1}{2} P_{k-1}\left(\operatorname{rot}_{k}\left(\Pi_{k}^{\perp} \underset{\sim}{u}\right)\right) \underset{\approx}{\chi}\right]-\operatorname{grad}_{k} \underset{\sim}{\zeta}:\left[-\frac{1}{2}\left(\operatorname{rot}_{k}\left(\Pi_{k}^{\perp} \underset{\sim}{u}\right)\right) \underset{\approx}{\chi}\right] d x\right| \\
& +\mid \int_{\Omega}\left[-\frac{1}{2} P_{k-1}\left(\operatorname{rot}_{k} \underset{\sim}{\zeta} \underset{\approx}{\chi}\right]: \operatorname{grad}_{k}\left(\Pi_{k}^{\perp} \underset{\sim}{u}\right)-\left[-\frac{1}{2}\left(\operatorname{rot}_{k} \underset{\sim}{\zeta} \underset{\approx}{\chi}\right]: \operatorname{grad}_{\approx}\left(\Pi_{k}^{\perp} \underset{\sim}{u}\right) d x \mid\right.\right. \\
& +\mid \int_{\Omega} \frac{1}{4}\left[P _ { k - 1 } \left(\operatorname{rot}_{k} \underset{\sim}{\zeta} \underset{\approx}{\chi}: \operatorname{rot}_{k}\left(\Pi_{k}^{\perp} \underset{\sim}{u} \underset{\approx}{\chi}\right]-\frac{1}{4}\left[\left(\operatorname{rot}_{k} \underset{\sim}{\zeta} \underset{\approx}{\chi}: \operatorname{rot}_{k}\left(\Pi_{k}^{\perp} \underset{\sim}{u} \underset{\approx}{\chi}\right] d x \mid .\right.\right.\right.\right.
\end{aligned}
$$

For the first term of (2.57) we have by (2.30) and (2.31)

$$
\begin{aligned}
& \left|\int_{\Omega} \frac{1}{2} \operatorname{grad}_{\approx} \zeta:\left(\operatorname{rot}_{k}\left(\Pi_{k}^{\perp} \underset{\sim}{u}\right)-P_{k-1} \operatorname{rot}_{k}\left(\Pi_{k}^{\perp} \underset{\sim}{u}\right)\right) \underset{\approx}{\chi} d x\right| \\
& =\left|\frac{1}{2} \int_{\Omega}\left(\operatorname{grad}_{\approx} \underset{\sim}{\zeta}-P_{k-1} \underset{\approx}{\operatorname{grad}_{k} \underset{\sim}{\zeta}}\right):\left(\operatorname{rot}_{k}\left(\Pi_{k}^{\perp} \underset{\sim}{u}\right)-P_{k-1} \operatorname{rot}_{k}\left(\Pi_{k}^{\perp} \underset{\sim}{u}\right)\right) \underset{\approx}{\chi \underset{\approx}{\chi}} d x\right|
\end{aligned}
$$

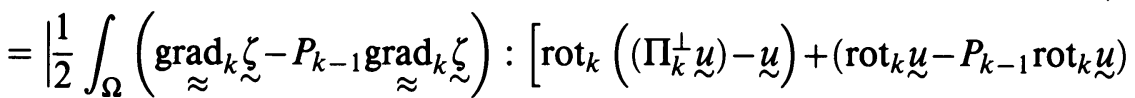

$$
\begin{aligned}
& \left.+P_{k-1}\left(\operatorname{rot}_{k}\left(\underset{\sim}{u}-\Pi_{k}^{\perp} \underset{\sim}{u}\right)\right) \underset{\approx}{\chi}\right] d x \\
& \leq\left. C h_{k}^{2} \underset{\sim}{\mid}\right|_{H^{2}(\Omega)} \underset{\sim}{\mid \underset{H^{2}(\Omega)}{\mid}} \\
& \leq C h_{k}^{2}\|\underset{\sim}{w}\|_{\mathcal{\sim}^{2}(\Omega)}\left(\|\underset{\sim}{f}\|_{{\underset{\sim}{L}}^{2}(\Omega)}+\sum_{i=1}^{n}\|\underset{\sim}{g}\|_{{\underset{\sim}{H}}^{1 / 2}\left(\Gamma_{i}\right)}\right) \text {. }
\end{aligned}
$$

Similar arguments yield the same bound for the second and third terms of (2.57).

Putting these bounds into (2.53), we have

$$
\begin{aligned}
& \left.\mid \mathscr{B}_{k}\left(\underset{\sim}{\zeta}-\underset{\sim}{\zeta}, \xi-\xi_{k}\right),\left(\Pi_{k}^{\perp} \underset{\sim}{\sim}, P_{k-1} p\right)\right) \mid \\
& \quad \leq C h_{k}^{2}\|\underset{\sim}{w}\|_{\mathcal{L}^{2}(\Omega)}\left(\|\underset{\sim}{f}\|_{\mathcal{L}^{2}(\Omega)}+\sum_{i=1}^{n}\|\underset{\sim}{g}\|_{H^{1 / 2}\left(\Gamma_{i}\right)}\right) .
\end{aligned}
$$

We can analogously (by interchanging $(\underset{\sim}{\mathcal{u}}, p)$ with $(\underset{\sim}{\zeta}, \xi)$, and $\left(\underset{\sim}{\mathcal{u}}, p_{k}\right)$ with $\left.\left({\underset{\sim}{\zeta}}_{k}, \xi_{k}\right)\right)$ derive the following estimate:

$$
\begin{aligned}
& \left|\mathscr{B}_{k}\left(\left(\Pi_{k}^{\perp} \underset{\sim}{\underset{\sim}{\zeta}}, P_{k-1} \xi\right),\left(\underset{\sim}{u}-{\underset{\sim}{u}}_{k}, p-p_{k}\right)\right)\right| \\
& \leq C h_{k}^{2}|\zeta|_{\mathcal{N}^{2}(\Omega)}|\underline{\sim}|_{H^{2}(\Omega)} \\
& \leq C h_{k}^{2}\|\underset{\sim}{w}\|_{\mathcal{\sim}^{2}(\Omega)}\left(\|f\|_{\mathcal{L}^{2}(\Omega)}+\sum_{i=1}^{n}\|\underset{\sim}{g}\|_{\mathcal{H}^{1 / 2}\left(\Gamma_{i}\right)}\right) \text {. }
\end{aligned}
$$


Finally, we have

$$
\begin{aligned}
& \left|\mathscr{B}((\underset{\sim}{\zeta}, \xi),(\underset{\sim}{u}, p))-\mathscr{B}_{k}((\underset{\sim}{\zeta}, \xi),(\underset{\sim}{u}, p))\right| \\
& =\left|\int_{\Omega} \underset{\approx k}{\epsilon} \underset{\sim}{(\zeta)}: \underset{\approx}{\epsilon}(\underset{\sim}{\mathcal{u}})-\underset{\approx k}{\epsilon^{*}}(\underset{\sim}{\zeta}): \underset{\approx k}{\epsilon}(\underset{\sim}{\mathcal{u}})\right| \\
& \leq C h_{k}^{2}\|\underset{\sim}{w}\|_{L^{2}(\Omega)}\left(\|f\|_{\mathcal{\sim}^{2}(\Omega)}+\sum_{i=1}^{n}\|\underset{\sim}{g}\|_{H^{1 / 2}\left(\Gamma_{i}\right)}\right),
\end{aligned}
$$

which is obtained by arguments similar to those in the proof of (2.56). Combining (2.51), (2.52), (2.58), (2.59), and (2.60), we therefore have

$$
\begin{aligned}
& \left|\frac{1}{2 \mu} \int_{\Omega}(\underset{\sim}{u}-\underset{\sim}{u}) \cdot \underset{\sim}{w} d x\right| \\
& \quad \leq C h_{k}^{2}\|\underset{\sim}{w}\|_{L^{2}(\Omega)}\left(\|\|_{\sim}^{f}\left\|_{L^{2}(\Omega)}+\sum_{i=1}^{n}\right\| \underset{\sim}{g} \|_{\sim^{1 / 2}\left(\Gamma_{i}\right)}\right)
\end{aligned}
$$

for all $\underset{\sim}{w} \in \underset{\sim}{L}{ }_{\perp}^{2}(\Omega)$. Combining (2.61) and (2.44) completes the proof.

Remark. The $L^{2}$ estimate in Theorem 2.10 is crucial in our proof of convergence of the multigrid method. This is one reason why $\underset{\sim}{V} \stackrel{\perp}{\text { is preferable over }}$ $\hat{V}_{k}$. The duality argument in the proof of Theorem 2.10 would not work for $\hat{\hat{V}}_{k}^{k}$ because condition $(1.17)$ is only satisfied by $(\underset{\sim}{w}, 0)$ where $\underset{\sim}{w} \in \underset{\sim}{L_{\perp}^{2}}$.

\section{INTERGRID TRANSFER OPERATORS AND MESH-DEPENDENT NORMS}

In this section we define the intergrid transfer operators and the meshdependent norms. The estimates involving the intergrid transfer operators will play an important role in the convergence analysis of the multigrid method. by

The coarse-to-fine operator $I_{k-1}^{k}: \underset{\sim}{V}{ }_{k-1} \times Q_{k-2} \longrightarrow \underset{\sim}{V} \times Q_{k-1}$ is defined

$$
I_{k-1}^{k}(\underset{\sim}{v}, q)=(\underset{\sim}{P} \underset{\sim}{v}, q)
$$

where $\underset{\sim}{P}: \underset{\sim}{L^{2}}(\Omega) \longrightarrow \underset{\sim}{V}$ is the $L^{2}$ orthogonal projection operator. Note that $\underset{\sim}{P} \underset{\sim}{v}$ can be explicitly defined by

$$
\begin{aligned}
& {\underset{\sim}{P}}_{k}(\underset{\sim}{v})\left(m_{e}\right) \\
& = \begin{cases}\underset{\sim}{v}\left(m_{e}\right) \quad \text { if } m_{e} \in \text { int } T \text { for some } T \in \mathscr{T}_{k-1} \text { or if } m_{e} \in \partial \Omega, \\
\frac{1}{\left|T_{1}\right|+\left|T_{2}\right|}\left(\left|T_{1}\right|\left[\left.\underline{\sim}\right|_{T_{1}}\left(m_{e}\right)\right]+\left|T_{2}\right|\left[\left.v\right|_{T_{2}}\left(m_{e}\right)\right]\right) & \text { if } e=T_{1} \cap T_{2} \text { for some } \\
& T_{1}, T_{2} \in \mathscr{T}_{k-1} .\end{cases}
\end{aligned}
$$

In order to define the fine-to-coarse operator $I_{k}^{k-1}$, we introduce the following mesh-dependent inner product:

$$
\left(\left({\underset{\sim}{v}}_{1}, q_{1}\right),\left({\underset{\sim}{v}}_{2}, q_{2}\right)\right)_{k}:=\left({\underset{\sim}{v}}_{1},{\underset{\sim}{v}}_{2}\right)_{L^{2}(\Omega)}+h_{k}^{2}\left(q_{1}, q_{2}\right)_{L^{2}(\Omega)}
$$

Then $I_{k}^{k-1}:{\underset{\sim}{V}}_{k} \times Q_{k-1} \longrightarrow \underset{\sim}{V}{ }_{k-1} \times Q_{k-2}$ is defined by

$$
\left(I_{k}^{k-1}\left({\underset{\sim}{v}}_{1}, q_{1}\right),\left({\underset{\sim}{v}}_{2}, q_{2}\right)\right)_{k-1}=\left(\left({\underset{\sim}{v}}_{1}, q_{1}\right), I_{k-1}^{k}\left({\underset{\sim}{v}}_{2}, q_{2}\right)\right)_{k}
$$


for all $\left(\underset{\sim}{v}, q_{1}\right) \in \underset{\sim}{V} \times Q_{k-1}$ and $\left(\underset{\sim}{v}, q_{2}\right) \in \underset{\sim}{V}{ }_{k-1} \times Q_{k-2}$.

Lemma 3.1. The following properties of $I_{k-1}^{k}$ and $I_{k}^{k-1}$ hold:

(i) $\underset{\sim}{\mathrm{RM}} \subseteq \underset{\sim}{V}$ for $k=1,2, \ldots$.

(ii) Given any $(\underset{\sim}{v}, q) \in \underset{\sim}{V} \times Q_{k-1}$, then $(\underset{\sim}{v}, q) \in \underset{\sim}{\underset{\sim}{\perp}} \times Q_{k-1}$ if and only if $((\underset{\sim}{v}, q),(\underset{\sim}{w}, 0))_{k}=0$ for all $\underset{\sim}{w} \in \mathbf{R M}$.

(iii) $I_{k-1}^{k}: \underset{\sim}{V} \underset{k-1}{\perp} \times Q_{k-2} \longrightarrow \underset{\sim}{\stackrel{\perp}{\perp}} \times Q_{k-1}$.

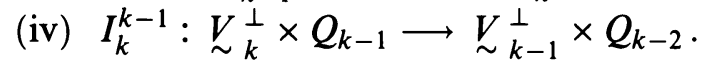

Proof. Property (i) is trivial. Property (ii) follows from (3.3), (iii) follows from (3.1), (i) and (ii), and (iv) follows from (3.1), (3.4), (i) and (ii).

Remark. The fact that the constraints on $\underset{\sim}{\underset{k}{\perp}}$ can be enforced by the meshdependent inner product as described in Lemma 3.1 (ii) is another reason why $\underset{\sim}{\sim} \underset{k}{\perp}$ is preferable to $\underset{\sim}{\hat{V}}$. As a consequence, these constraints are preserved by the intergrid transfer operators $I_{k-1}^{k}$ and $I_{k}^{k-1}$ (Lemmas 3.1 (iii) and (iv)). We will take advantage of this in the construction of the multigrid algorithm in $\S 4$.

Let $B_{k}: \underset{\sim}{V} \times Q_{k-1} \longrightarrow \underset{\sim}{V} \times Q_{k-1}$ be defined by

$$
\begin{aligned}
& \left(B_{k}\left({\underset{\sim}{1}}_{1}^{v}, q_{1}\right),\left(\underset{\sim}{v}, q_{2}\right)\right)_{k} \\
& \quad=\mathscr{B}_{k}\left(\left({\underset{\sim 1}{v}}_{1}^{v}, q_{1}\right),\left({\underset{\sim}{\sim}}_{2}^{v}, q_{2}\right)\right) \quad \forall\left({\underset{\sim}{v}}_{1}^{v}, q_{1}\right),\left({\underset{\sim}{2}}_{2}^{v}, q_{2}\right) \in \underset{\sim k}{V} \times Q_{k-1} .
\end{aligned}
$$

Lemma 3.2. The operator $B_{k}$ maps $\underset{\sim}{V} \times Q_{k-1}$ into $\underset{\sim}{V} \underset{k}{\perp} \times Q_{k-1}$.

Proof. Let $(\underset{\sim}{v}, q) \in \underset{\sim}{V}{ }_{k} \times Q_{k-1}$. Then

$$
\left(B_{k}(\underset{\sim}{v}, q),(\underset{\sim}{w}, 0)\right)_{k}=\mathscr{B}_{k}((\underset{\sim}{v}, q),(\underset{\sim}{w}, 0))=0 \quad \forall \underset{\sim}{w} \in \mathbf{R M} .
$$

Therefore, $B_{k}(\underset{\sim}{v}, q) \in \underset{\sim}{\underset{1}{\perp}} \times Q_{k-1}$ by Lemma 3.1 (ii).

Let $B_{k}^{\perp}: \underset{\sim}{V} \underset{k}{\perp} \times Q_{k-1} \longrightarrow \underset{\sim}{V} \underset{k}{\perp} \times Q_{k-1}$ be the restriction of $B_{k}$ to $\underset{\sim}{\underset{\perp}{\perp}} \times Q_{k-1}$.

Lemma 3.3. There exists a positive constant $C$ such that the spectral radius of $B_{k}^{\perp}$ is less than or equal to $C h_{k}^{-2}$ for $k=1,2, \ldots$.

Proof. This is an immediate consequence of (3.3), (3.5), (1.29) and the following inverse estimate (cf. [4, Theorem 3.2.6]):

$$
\|\underset{\sim}{w}\|_{k} \leq C h_{k}^{-1}\|\underset{\sim}{w}\|_{\mathcal{\sim}^{2}(\Omega)} \quad \forall \underset{\sim}{w} \in \underset{\sim}{V}
$$

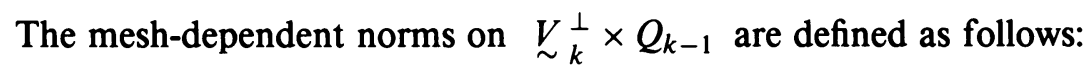

$$
\|(\underset{\sim}{v}, q)\|_{s, k}:=\sqrt{\left(\left(B_{k}^{\perp}\right)^{s / 2}(\underset{\sim}{v}, q),(\underset{\sim}{v}, q)\right)_{k}} \quad \forall(\underset{\sim}{v}, q) \in \underset{\sim}{V_{k}^{\perp}} \times Q_{k-1} .
$$

Since $B_{k}^{\perp}$ is nonsingular (cf. Proposition 2.7) and symmetric, $\left(B_{k}^{\perp}\right)^{2}$ is positive definite and (3.7) defines a norm on $\underset{\sim}{\underset{1}{\perp}} \times Q_{k-1}$ for each $s \in \mathbb{R}$. 
The following properties of the mesh-dependent norms are trivial:

$$
\|(\underset{\sim}{v}, q)\|_{0, k}=\sqrt{\|\underset{\sim}{v}\|_{\mathcal{L}^{2}(\Omega)}^{2}+h_{k}^{2}\|q\|_{L^{2}(\Omega)}^{2}} \forall(\underset{\sim}{v}, q) \in \underset{\sim}{\underset{\sim}{V}} \times Q_{k-1}^{\perp},
$$$$
\left.\mid \mathscr{B}_{k}\left(\left(\underset{\sim 1}{v}, q_{1}\right), \underset{\sim}{v}, q_{2}\right)\right) \mid
$$

$$
\leq\left\|\left(\underset{\sim}{v}, q_{1}\right)\right\|_{2, k}\left\|\left(\underset{\sim}{v}, q_{2}\right)\right\|_{0, k} \quad \forall\left(\underset{\sim}{v}, q_{1}\right),\left(\underset{\sim}{v}, q_{2}\right) \in \underset{\sim}{V_{k}^{\perp}} \times Q_{k-1},
$$

and

$$
\|(\underset{\sim}{v}, q)\|_{2, k}=\sup _{\left(\sim_{\sim}^{\prime}, q^{\prime}\right) \in \underset{\sim}{\perp} \times Q_{k-1} \backslash\{(\underset{\sim}{0}, 0)\}} \frac{\left|\mathscr{B}_{k}\left((\underset{\sim}{v}, q),\left({\underset{\sim}{v}}^{\prime}, q^{\prime}\right)\right)\right|}{\left\|\left({\underset{\sim}{v}}^{\prime}, q^{\prime}\right)\right\|_{0, k}}
$$

for all $(\underset{\sim}{v}, q) \in \underset{\sim}{V} \stackrel{\perp}{k} \times Q_{k-1}$.

Lemma 3.4. There exists a positive constant $C$ such that

(i) $\left\|I_{k-1}^{k}(\underset{\sim}{v}, q)\right\|_{0, k} \leq C\|(\underset{\sim}{v}, q)\|_{0, k-1}$ for all $(\underset{\sim}{v}, q) \in \underset{\sim}{V} \underset{k-1}{\perp} \times Q_{k-2}$.

(ii) $\|\underset{\sim}{v}-\underset{\sim}{P} \underset{\sim}{v}\|_{\sim_{\sim}^{2}(\Omega)} \leq C h_{k}\|\underset{\sim}{v}\|_{k-1}$ for all $\underset{\sim}{v} \in \underset{\sim}{V} \underset{k-1}{\perp}$.

(iii) $\left\|I_{k-1}^{k}\left(\Pi_{k-1}^{\perp} \underset{\sim}{\phi}, P_{k-2} \psi\right)-\left(\Pi_{k}^{\perp} \underset{\sim}{\phi}, P_{k-1} \psi\right)\right\|_{0, k} \leq C h_{k}^{2}\left\{\left.\left|\underset{\sim}{\mid \underset{\sim}{\left.\right|^{2}(\Omega)}}+\right| \psi\right|_{H^{1}(\Omega)}\right\}$ for all $(\underset{\sim}{\phi}, \psi) \in \underset{\sim}{H_{\perp}}(\Omega) \times H^{1}(\Omega)$.

Proof. Since $P_{k}$ is the $L^{2}$ orthogonal projection onto $\underset{\sim}{V}$, the estimate (i) follows from (3.1) and (3.8). The proof of inequality (ii) is based on the averaging formula (3.2), and a straightforward computation. For more details we refer the reader to the proof of Theorem 2.3 in [2]. Estimate (iii) is a consequence of (3.8) and the interpolation error estimates (2.30) and (2.31).

Let $P_{k}^{k-1}: \underset{\sim}{V} \underset{k}{\perp} \times Q_{k-1} \longrightarrow \underset{\sim}{\underset{1}{\perp}} \times Q_{k-2}$ be defined by

$$
\mathscr{B}_{k-1}\left(P_{k}^{k-1}\left({\underset{\sim}{v}}_{1}^{v}, q_{1}\right),\left(\underset{\sim}{v}, q_{2}\right)\right)=\mathscr{B}_{k}\left(\left({\underset{\sim}{v}}_{1}, q_{1}\right), I_{k-1}^{k}\left(\underset{\sim}{v}, q_{2}\right)\right)
$$

for all $\left(\underset{\sim}{v}, q_{1}\right) \in \underset{\sim}{V} \underset{k}{\perp} \times Q_{k-1}$ and $\left(\underset{\sim}{v}, q_{2}\right) \in \underset{\sim}{V} \underset{k-1}{\perp} \times Q_{k-2}$. The operator $P_{k}^{k-1}$ will appear in the convergence analysis but not in the multigrid algorithm.

It follows from Lemma 3.4 (i) and (3.9)-(3.11) that

$$
\left\|P_{k}^{k-1}(\underset{\sim}{v}, q)\right\|_{2, k-1} \leq C\|(\underset{\sim}{v}, q)\|_{2, k} \quad \forall(\underset{\sim}{v}, q) \in \underset{\sim}{V} \underset{k}{\perp} \times Q_{k-1} .
$$

The next lemma is the basis of Lemmas 3.6 and 3.7, which are crucial for the proof of the approximation property (Lemma S.3) in the Supplement.

Lemma 3.5. Let $k \geq 2$ and $\underset{\sim}{w} \in \underset{\sim}{L}(\Omega)$. Assume that $\left(\underset{\sim}{\zeta}, \xi_{k}\right) \in \underset{\sim}{V} \underset{k}{\perp} \times Q_{k-1}$ satisfies

$$
\mathscr{B}_{k}\left(\left(\underset{\sim}{\zeta}, \xi_{k}\right),(\underset{\sim}{v}, q)\right)=\int_{\Omega} \underset{\sim}{w} \cdot \underset{\sim}{v} d x \quad \forall(\underset{\sim}{v}, q) \in \underset{\sim}{V} \stackrel{\perp}{x} \times Q_{k-1},
$$

and $\left({\underset{\sim}{\zeta}}_{k-1}, \xi_{k-1}\right) \in \underset{\sim}{V} \underset{k-1}{\perp} \times Q_{k-2}$ satisfies

$$
\mathscr{B}_{k-1}\left(\left({\underset{\sim}{k-1}}_{k-1}, \xi_{k-1}\right),(\underset{\sim}{v}, q)\right)=\int_{\Omega} \underset{\sim}{w} \cdot \underset{\sim}{v} d x \quad \forall(\underset{\sim}{v}, q) \in \underset{\sim}{\underset{k-1}{\perp}} \times Q_{k-2} .
$$


Then there exists a positive constant $C$ such that

$$
\left\|\left({\underset{\sim}{\zeta}}_{k-1}, \xi_{k-1}\right)-P_{k}^{k-1}\left(\underset{\sim}{\zeta}, \xi_{k}\right)\right\|_{0, k-1} \leq C h_{k}^{2}\|\underset{\sim}{w}\|_{L^{2}(\Omega)} .
$$

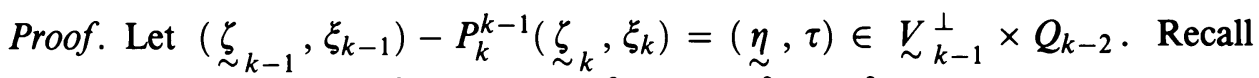
that by (3.8), $\|(\underset{\sim}{\eta}, \tau)\|_{0, k-1}^{2}=\|\underset{\sim}{\eta}\|_{L^{2}(\Omega)}^{2}+h_{k-1}^{2}\|\tau\|_{L^{2}(\Omega)}^{2}$. We will estimate $\|\underset{\sim}{\eta}\|_{\mathcal{\sim}^{2}(\Omega)}$ and $\underset{h_{k-1}}{\sim}\|\tau\|_{L^{2}(\Omega)}$ by two duality arguments.

Since $\underset{\sim}{\eta} \in \underset{\sim}{L} \underset{\perp}{2}(\Omega)$, the boundary value problem

$$
-\operatorname{div}\{2 \mu \underset{\sim}{\epsilon}(\phi)+\lambda \operatorname{tr}(\underset{\sim}{\epsilon} \underset{\sim}{\phi})) \underset{\approx}{\delta}\}=2 \mu \underset{\sim}{\underset{\sim}{\gamma}} \text { in } \Omega
$$

$$
(2 \mu \underset{\sim}{\epsilon}(\underset{\sim}{\phi})+\lambda \operatorname{tr}(\underset{\sim}{\epsilon}(\phi)) \underset{\sim}{\delta}) \underset{\sim}{\nu_{i}} \mid \Gamma_{i}=0, \quad 1 \leq i \leq n,
$$

has a unique solution $\underset{\sim}{\phi} \in \underset{\sim}{H}{ }_{\perp}^{2}(\Omega)$ because (1.16) and (1.17) are satisfied. The elliptic regularity estimate (1.20) implies that

$$
\|\underset{\sim}{\phi}\|_{\mathcal{\sim}^{2}(\Omega)} \leq C\|\underset{\sim}{\eta}\|_{\mathcal{\sim}^{2}(\Omega)}
$$

Let $\left({\underset{\sim}{\phi-1}}_{k-1}, \psi_{k-1}\right) \in \underset{\sim}{\underset{k-1}{\perp}} \times Q_{k-2}$ satisfy

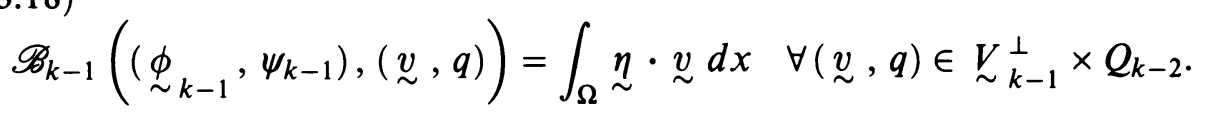

By Theorem 2.10, we have

$$
\left\|\underset{\sim}{\phi}-\underset{\sim}{\phi}{ }_{k-1}\right\|_{L^{2}(\Omega)} \leq C h_{k}^{2}\|\underset{\sim}{\eta}\|_{L^{2}(\Omega)}
$$

But by (3.18), (3.11), the definition of $(\underset{\sim}{\eta}, \tau),(3.13),(3.14),(3.19),(3.17)$, and $(2.30)$, we have

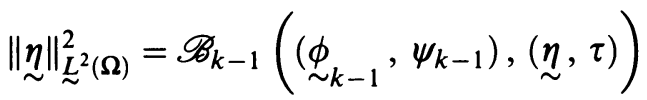

$$
\begin{aligned}
& =\mathscr{B}_{k-1}\left(\left(\underset{\sim k-1}{\phi}, \psi_{k-1}\right),\left(\underset{\sim}{\zeta_{k-1}}, \xi_{k-1}\right)\right) \\
& -\mathscr{B}_{k}\left(I_{k-1}^{k}\left(\underset{\sim}{\phi} \underset{k-1}{\phi}, \psi_{k-1}\right),\left({\underset{\sim}{k}}_{k}, \xi_{k}\right)\right) \\
& =\int_{\Omega} \underset{\sim}{w} \cdot(\underset{\sim k-1}{\phi}-\underset{\sim k}{P} \underset{\sim k-1}{\phi}) d x \\
& \leq\|\underset{\sim}{w}\|_{\mathcal{\sim}^{2}(\Omega)}\left(\|\underset{\sim}{\phi}-\underset{\sim}{\phi}\|_{\mathcal{\sim}^{2}(\Omega)}+\left\|\underset{\sim}{\phi}-\Pi_{k}^{\perp} \underset{\sim}{\perp}\right\|_{\mathcal{\sim}^{2}(\Omega)}\right. \\
& \left.+\left\|\sim_{k}\left(\Pi_{k}^{\perp} \underset{\sim}{\phi}-\underset{\sim k-1}{\phi}\right)\right\|_{\mathcal{\sim}^{2}(\Omega)}\right) \\
& \leq\|\underset{\sim}{w}\|_{L^{2}(\Omega)}\left(C h_{k}^{2}\|\underset{\sim}{\|}\|_{L^{2}(\Omega)}\right) .
\end{aligned}
$$

Therefore,

$$
\|\underset{\sim}{\eta}\|_{\sim^{2}(\Omega)} \leq C h_{k}^{2}\|\underset{\sim}{w}\|_{\sim^{2}(\Omega)} .
$$




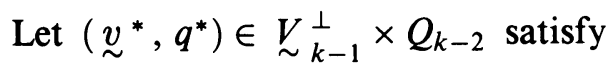

(3.21) $\mathscr{B}_{k-1}\left(\left({\underset{\sim}{v}}^{*}, q^{*}\right),(\underset{\sim}{v}, q)\right)=h_{k-1}^{2} \int_{\Omega} \tau q d x \quad \forall(\underset{\sim}{v}, q) \in \underset{\sim}{\stackrel{\perp}{\perp} \times Q_{k-2}}$

By Proposition 2.7 we have

$$
\left\|\stackrel{\sim}{\sim}^{*}\right\|_{k-1}+\left\|q^{*}\right\|_{L^{2}(\Omega)} \leq h_{k-1}^{2}\|\tau\|_{L^{2}(\Omega)}
$$

From Lemma 3.4 (ii), (3.21) and (3.22), we have

$$
\begin{aligned}
h_{k-1}^{2}\|\tau\|_{L^{2}(\Omega)}^{2} & \left.=\mathscr{B}_{k-1}\left(\left({\underset{\sim}{v}}^{*}, q^{*}\right), \underset{\sim}{\eta}, \tau\right)\right) \\
& =\mathscr{B}_{k-1}\left(\left({\underset{\sim}{v}}^{*}, q^{*}\right),\left(\underset{\sim}{\underset{\sim}{\zeta}-1}, \xi_{k-1}\right)\right)-\mathscr{B}_{k}\left(I_{k-1}^{k}\left({\underset{\sim}{v}}^{*}, q^{*}\right),\left(\underset{\sim}{\zeta}, \xi_{k}\right)\right) \\
& =\int_{\Omega} \underset{\sim}{w} \cdot\left({\underset{\sim}{v}}^{*}-\underset{\sim}{P} \underset{\sim}{v^{*}}\right) d x \\
& \leq\|\underset{\sim}{w}\|_{\mathcal{\sim}^{2}(\Omega)}\left\|{\underset{\sim}{v}}^{*}-\underset{\sim}{P} \underset{\sim}{v^{*}}\right\|_{L_{\sim}^{2}(\Omega)} \\
& \leq C h_{k}^{3}\|\underset{\sim}{w}\|_{\mathcal{\sim}^{2}(\Omega)}\|\tau\|_{L^{2}(\Omega)} .
\end{aligned}
$$

Hence,

$$
h_{k-1}\|\tau\|_{L^{2}(\Omega)} \leq C h_{k}^{2}\|\underset{\sim}{w}\|_{L^{2}(\Omega)}
$$

The lemma now follows from (3.20) and (3.23).

Lemma 3.6. There exists a positive constant $C$ such that for $k \geq 2$

$$
\|\underset{\sim}{v}-\underset{\sim}{P} \underset{\sim}{\sim}\|_{L^{2}(\Omega)} \leq C h_{k}^{2}\|(\underset{\sim}{v}, q)\|_{2, k-1}
$$

for all $(\underset{\sim}{v}, q) \in \underset{\sim}{\underset{k-1}{\perp}} \times Q_{k-2}$.

Proof. Given any $(\underset{\sim}{v}, q) \in \underset{\sim}{\underset{\sim}{\perp}} \stackrel{\perp}{k-1} \times Q_{k-2}$, let $\left(\underset{\sim}{\zeta}, \xi_{k}\right) \in \underset{\sim}{V} \underset{k}{\perp} \times Q_{k-1}$ satisfy

$$
\begin{aligned}
\mathscr{B}_{k} & \left(\left({\underset{\sim}{\zeta}}_{k}, \xi_{k}\right),\left({\underset{\sim}{v}}^{\prime}, q^{\prime}\right)\right) \\
& =\int_{\Omega}(\underset{\sim}{v}-\underset{\sim}{P} \underset{\sim}{v}) \cdot{\underset{\sim}{v}}^{\prime} d x \quad \forall\left({\underset{\sim}{v}}^{\prime}, q^{\prime}\right) \in{\underset{\sim}{L}}_{k}^{\perp} \times Q_{k-1}
\end{aligned}
$$

and $\left({\underset{\sim}{\zeta}}_{k-1}, \xi_{k-1}\right) \in \underset{\sim}{V} \stackrel{\perp}{k-1} \times Q_{k-2}$ satisfy

$$
\begin{aligned}
\mathscr{B}_{k-1} & \left.\left(\underset{\sim}{(\underset{\sim}{\zeta}, 1}, \xi_{k-1}\right),\left({\underset{\sim}{v}}^{\prime}, q^{\prime}\right)\right) \\
= & \int_{\Omega}(\underset{\sim}{v}-\underset{\sim}{P} \underset{\sim}{v}) \cdot{\underset{\sim}{v}}^{\prime} d x \quad \forall\left({\underset{\sim}{v}}^{\prime}, q^{\prime}\right) \in{\underset{\sim}{\sim}}_{k-1}^{\perp} \times Q_{k-2} .
\end{aligned}
$$

Therefore, using (3.1), (3.11), and Lemma 3.5, we have

$$
\begin{aligned}
\|\underset{\sim}{v}-\underset{\sim}{P} \underset{\sim}{v}\|_{L^{2}(\Omega)}^{2} & =\int_{\Omega}(\underset{\sim}{v}-\underset{\sim}{P} \underset{\sim}{v}) \cdot \underset{\sim}{v} d x-\int_{\Omega}(\underset{\sim}{v}-\underset{\sim}{P} \underset{\sim}{v}) \cdot \underset{\sim}{P} \underset{\sim}{v} d x \\
& =\mathscr{B}_{k-1}\left(\left({\underset{\sim}{k-1}}_{k}, \xi_{k-1}\right),(\underset{\sim}{v}, q)\right)-\mathscr{B}_{k}\left(\left(\underset{\sim}{\zeta}, \xi_{k}\right), I_{k-1}^{k}(\underset{\sim}{v}, q)\right) \\
& =\mathscr{B}_{k-1}\left(\left({\underset{\sim}{k-1}}_{k-1}, \xi_{k-1}\right)-P_{k}^{k-1}\left(\underset{\sim}{\zeta}, \xi_{k}\right),(\underset{\sim}{v}, q)\right) \\
& \leq\left\|\left({\underset{\sim}{k-1}}_{k-1}, \xi_{k-1}\right)-P_{k}^{k-1}\left(\underset{\sim}{\zeta}, \xi_{k}\right)\right\|_{0, k-1}\|(\underset{\sim}{v}, q)\|_{2, k-1} \\
& \leq C h_{k}^{2}\|\underset{\sim}{v}-\underset{\sim}{P} \underset{\sim}{v}\|_{L^{2}(\Omega)}\|(\underset{\sim}{v}, q)\|_{2, k-1} .
\end{aligned}
$$


Hence,

$$
\|\underset{\sim}{v}-\underset{\sim}{P} \underset{\sim}{\sim}\|_{L^{2}(\Omega)} \leq C h_{k}^{2}\|(\underset{\sim}{v}, q)\|_{2, k-1}
$$

Lemma 3.7. Let $k \geq 2$ and $w \in L^{2}(\Omega),\left(\underset{\sim}{v}, q_{k}\right) \in \underset{\sim}{V} \underset{k}{\perp} \times Q_{k-1}$ satisfy

$$
\mathscr{B}_{k}\left(\left({\underset{\sim}{v}}_{k}, q_{k}\right),(\underset{\sim}{v}, q)\right)=\int_{\Omega} w q d x \quad \forall(\underset{\sim}{v}, q) \in \underset{\sim}{V_{k}^{\perp}} \times Q_{k-1},
$$

and $\left({\underset{\sim}{v}}_{k-1}, q_{k-1}\right) \in \underset{\sim}{\underset{k-1}{\perp}} \times Q_{k-2}$ satisfy

(3.28) $\mathscr{B}_{k-1}\left(\left({\underset{\sim}{v}}_{k-1}, q_{k-1}\right),(\underset{\sim}{v}, q)\right)=\int_{\Omega} w q d x \quad \forall(\underset{\sim}{v}, q) \in \underset{\sim}{V_{k-1}^{\perp}} \times Q_{k-2}$.

Then there exists a positive constant $C$ such that

$$
\left\|\left({\underset{\sim}{v}}_{k}, q_{k}\right)-I_{k-1}^{k}\left({\underset{\sim}{v}}_{k-1}, q_{k-1}\right)\right\|_{0, k} \leq C h_{k}\|w\|_{L^{2}(\Omega)} .
$$

Proof. Recall that

$$
\begin{aligned}
& \left\|\left({\underset{\sim}{v}}_{k}, q_{k}\right)-I_{k-1}^{k}\left({\underset{\sim}{v}}_{k-1}^{v}, q_{k-1}\right)\right\|_{0, k}^{2} \\
& \quad=\|\underset{\sim k}{v}-\underset{\sim k}{\underset{\sim}{v}} \underset{k-1}{v}\|_{L^{2}(\Omega)}^{2}+h_{k}^{2}\left\|q_{k}-q_{k-1}\right\|_{L^{2}(\Omega)}^{2} .
\end{aligned}
$$

We first estimate $\left\|{\underset{\sim}{v}}_{k}-\underset{\sim}{P}{\underset{\sim}{v}}_{k-1}^{v}\right\|_{L^{2}(\Omega)}$ by a duality argument.

Since ${\underset{\sim}{v}}_{k}-\underset{\sim}{P} \underset{\sim}{v} \underset{k-1}{v} \in \underset{\sim}{L} \underset{\perp}{2}(\Omega)$, the boundary value problem

$$
\begin{aligned}
& -\operatorname{div}\{2 \mu \underset{\sim}{\epsilon}(\zeta)+\lambda \operatorname{tr}(\underset{\sim}{\epsilon}(\zeta)) \underset{\approx}{\delta}\}=2 \mu(\underset{\sim}{v}-\underset{\sim}{P} \underset{\sim}{v} \underset{\sim}{v}) \text { in } \Omega \text {, } \\
& (2 \mu \underset{\sim}{\epsilon}(\zeta)+\lambda \operatorname{tr}(\underset{\sim}{\epsilon}(\zeta)) \underset{\sim}{\delta}) \underset{\sim}{\nu_{i}} \mid \Gamma_{i}=0, \quad 1 \leq i \leq n,
\end{aligned}
$$

has a unique solution $\underset{\sim}{\zeta} \underset{\sim}{H}{ }_{\perp}^{2}(\Omega)$ because (1.16) and (1.17) are satisfied.

Let $\left(\underset{\sim}{\zeta}, \xi_{k}\right) \in \underset{\sim}{V} \underset{k}{\perp} \times Q_{k-1}$ satisfy

$\mathscr{B}_{k}\left(\left(\underset{\sim}{\zeta}, \xi_{k}\right),(\underset{\sim}{v}, q)\right)=\int_{\Omega}\left({\underset{\sim}{v}}_{k}-\underset{\sim}{P} \underset{\sim}{v}{ }_{k-1}\right) \cdot \underset{\sim}{v} d x \quad \forall(\underset{\sim}{v}, q) \in{\underset{\sim}{V}}_{k}^{\perp} \times Q_{k-1}$,

and $\left({\underset{\sim}{\zeta}}_{k-1}, \xi_{k-1}\right) \in \underset{\sim}{V} \underset{k-1}{\perp} \times Q_{k-2}$ satisfy

$$
\begin{aligned}
\mathscr{B}_{k-1} & \left.\left(\left({\underset{\sim}{\zeta k-1}}_{\zeta_{k-1}}, \xi_{k}\right), \underset{\sim}{v}, q\right)\right) \\
= & \int_{\Omega}(\underset{\sim k}{v}-\underset{\sim k}{P} \underset{\sim k-1}{v}) \cdot \underset{\sim}{v} d x \quad \forall(\underset{\sim}{v}, q) \in{\underset{\sim k}{k-1}}^{\perp} \times Q_{k-2} .
\end{aligned}
$$

By Theorem 2.9 and the elliptic regularity estimate (1.20) we have

$$
\begin{aligned}
& \left\|\xi_{k}-\xi_{k-1}\right\|_{L^{2}(\Omega)} \leq\left\|\xi_{k}-\frac{\lambda}{2 \mu} \operatorname{div} \underset{\sim}{\zeta}\right\|_{L^{2}(\Omega)}+\left\|\xi_{k-1}-\frac{\lambda}{2 \mu} \operatorname{div} \underset{\sim}{\zeta}\right\|_{L^{2}(\Omega)} \\
& \leq C h_{k}\|\underset{\sim}{v}-\underset{\sim}{P} \underset{\sim}{\underset{v}{v}}\|_{k-1} \|_{L^{2}(\Omega)} \text {. }
\end{aligned}
$$


Let $(\underset{\sim}{\eta}, \tau)=\left(\underset{\sim}{\zeta}, \xi_{k}\right)-P_{k}^{k-1}\left({\underset{\sim}{\zeta}}_{k}, \xi_{k}\right)$. Using (3.1), (3.11), (3.27), and (3.28), we have

$$
\begin{aligned}
\| \sim_{k} & -\underset{\sim}{P} \underset{\sim}{\underset{\sim}{v}}{ }_{k-1} \|_{\mathcal{L}^{2}(\Omega)}^{2} \\
& =\mathscr{B}_{k}\left(\left(\underset{\sim}{\zeta}, \xi_{k}\right),\left({\underset{\sim}{v}}_{k}, q_{k}\right)-I_{k-1}^{k}\left({\underset{\sim}{v-1}}_{k-1}^{v}, q_{k-1}\right)\right) \\
& =\mathscr{B}_{k}\left(\left({\underset{\sim}{\zeta k}}_{k}, \xi_{k}\right),\left({\underset{\sim}{v}}_{k}, q_{k}\right)\right)-\mathscr{B}_{k-1}\left(P_{k}^{k-1}\left({\underset{\sim}{k}}_{k}, \xi_{k}\right),\left({\underset{\sim}{k-1}}_{k}, q_{k-1}\right)\right) \\
& =\int_{\Omega} w \tau d x \\
& \leq\|w\|_{L^{2}(\Omega)}\|\tau\|_{L^{2}(\Omega)} .
\end{aligned}
$$

Since $(\underset{\sim}{\eta}, \tau)=\left({\underset{\sim}{\zeta}}_{k}, \xi_{k}\right)-\left({\underset{\sim}{\zeta}}_{k-1}, \xi_{k-1}\right)+\left(\left({\underset{\sim}{\zeta}}_{k-1}, \xi_{k-1}\right)-P_{k}^{k-1}\left({\underset{\sim}{\zeta}}_{k}, \xi_{k}\right)\right)$, it follows from (3.8), (3.30), and Lemma 3.5 that

$$
\begin{aligned}
\|\tau\|_{L^{2}(\Omega)} & \leq\left\|\xi_{k}-\xi_{k-1}\right\|_{L^{2}(\Omega)}+\frac{1}{h_{k}}\left\|\left({\underset{\sim}{\sim} k-1}_{\zeta_{k-1}}, \xi_{k-1}\right)-P_{k}^{k-1}\left(\underset{\sim}{\zeta_{k}}, \xi_{k}\right)\right\|_{0, k-1} \\
& \leq C h_{k}\|\|_{\sim}-\underset{\sim}{P} \underset{\sim}{\underset{v}{v}} \|_{L^{2}(\Omega)} .
\end{aligned}
$$

The estimates (3.31) and (3.32) together imply that

$$
\left\|{\underset{\sim}{v}}_{k}-\underset{\sim}{P} \underset{\sim}{\sim} \underset{k-1}{v}\right\|_{L^{2}(\Omega)} \leq C h_{k}\|w\|_{L^{2}(\Omega)} .
$$

On the other hand, Proposition 2.7 implies that

$$
\left\|{\underset{\sim}{v-1}}_{k-1}\right\|_{k-1}+\left\|q_{k-1}\right\|_{L^{2}(\Omega)} \leq C\|w\|_{L^{2}(\Omega)}
$$

and

$$
\left\|{\underset{\sim}{v}}_{k}\right\|_{k}+\left\|q_{k}\right\|_{L^{2}(\Omega)} \leq C\|w\|_{L^{2}(\Omega)}
$$

Therefore, we have

$$
h_{k}\left\|q_{k}-q_{k-1}\right\|_{L^{2}(\Omega)} \leq C h_{k}\|w\|_{L^{2}(\Omega)} .
$$

The estimate (3.29) now follows from (3.33) and (3.36).

\section{The MUlTigrid ALGORITHM}

We first describe the $k$ th-level iteration scheme. The full multigrid algorithm consists of a nested iteration of these schemes.

The $k$ th-level iteration. The kth-level iteration with initial guess $\left(\underset{\sim}{y}, z_{0}\right) \in$ $\underset{\sim}{V} \underset{k}{\perp} \times Q_{k-1}$ yields $M G\left(k,\left(\underset{\sim}{y}, z_{0}\right),(\underset{\sim}{w}, r)\right)$ as an approximate solution to the following equation in $\underset{\sim}{V} \underset{k}{\perp} \times Q_{k-1}$ :

$$
B_{k}^{\perp}(\underset{\sim}{y}, z)=(\underset{\sim}{w}, r) \text {. }
$$

For $k=1, M G\left(1,\left(\underset{\sim}{y}, z_{0}\right),(\underset{\sim}{w}, r)\right)$ is the solution obtained from a direct method. In other words,

$$
M G\left(1,\left({\underset{\sim}{y}}_{0}, z_{0}\right),(\underset{\sim}{w}, r)\right)=\left(B_{1}^{\perp}\right)^{-1}(\underset{\sim}{w}, r) .
$$


For $k>1$, there are two steps:

Smoothing step. Let $\left(\underset{\sim}{y_{l}}, z_{l}\right) \in \underset{\sim}{V} \times Q_{k-1}$ be defined recursively by the equations

$$
\begin{aligned}
\left(\underset{\sim l}{y}, z_{l}\right)= & \left({\underset{\sim}{l-1}}_{l}^{y}, z_{l-1}\right) \\
& +\frac{1}{\Lambda_{k}} B_{k}\left((\underset{\sim}{w}, r)-B_{k}\left({\underset{\sim}{l-1}}_{l-1}, z_{l-1}\right)\right), 1 \leq l \leq m,
\end{aligned}
$$

where $m$ is a positive integer independent of the mesh parameter $k$ and the Lamé constants $(\mu, \lambda)$, and $\Lambda_{k}:=C h_{k}^{-4}$ (cf. Lemma 3.3) dominates the spectral radius of $\left(B_{k}^{\perp}\right)^{2}$.

Correction step. Let $(\underset{\sim}{\bar{w}}, \bar{r}):=I_{k}^{k-1}\left((\underset{\sim}{w}, r)-B_{k}\left({\underset{\sim}{w}}_{m}, z_{m}\right)\right)$. Let $\left(\underset{\sim}{v}, q_{i}\right)$ $\in{\underset{\sim}{V-1}}_{V_{k-2}} \times Q_{i \leq 2)}(0 \leq i \leq$ defined recursively by

$$
\begin{aligned}
& \left({\underset{\sim}{0}}_{0}^{v}, q_{0}\right)=(\underset{\sim}{0}, 0) \text { and } \\
& \left({\underset{\sim}{v}}_{i}, q_{i}\right)=M G\left(k-1,\left({\underset{\sim}{v i-1}}_{i-1}, q_{i-1}\right),(\underset{\sim}{w}, \bar{r})\right), \quad i=1,2 .
\end{aligned}
$$

Then $M G\left(k,\left({\underset{\sim}{0}}_{0}^{y}, z_{0}\right),(\underset{\sim}{w}, g)\right)$ is defined to be $\left(\underset{\sim m}{y}, z_{m}\right)+I_{k-1}^{k}\left(\underset{\sim}{v}, q_{2}\right)$.

Remark. In the smoothing step we use $B_{k}$ instead of $B_{k}^{\perp}$ for the following reason. The space $V_{k} \times Q_{k-1}$ has a natural coordinate system, namely the values of $\underset{\sim}{v}$ at the midpoints of $\mathscr{T}_{k}$ and the values of $q$ on the triangles of $\mathscr{T}_{k-1}$, with respect to which $(\cdot, \cdot)_{k}$ is represented by a diagonal matrix, and $B_{k}$ is represented by a sparse banded matrix. In view of Lemma 3.2, the result of the smoothing step $\left(\underset{\sim}{y_{m}}, z_{m}\right)$ belongs to $\underset{\sim}{V}{ }_{k}^{\perp} \times Q_{k-1}$ automatically. Furthermore, the result of the correction step also belongs to $\underset{\sim}{V} \underset{k}{\perp} \times Q_{k-1}$ by Lemma 3.1 (iii) and (iv). Therefore, in the actual implementation of the multigrid method we use only the natural coordinate system of $\underset{\sim}{V} \times Q_{k-1}$ for $k>1$. We only have to handle the constraints of the $\underset{\sim}{V} \underset{k}{\perp}$ spaces at the coarsest level, namely when $k=1$.

The full multigrid algorithm. In the case $k=1$, the approximate solution $\left({\underset{\sim}{u}}_{1}^{*}, p_{1}^{*}\right)$ of $(1.26)$ is obtained by a direct method. The approximate solutions $\left({\underset{\sim}{u}}_{k}^{*}, p_{k}^{*}\right)(k \geq 2)$ of $(1.26)$ are obtained recursively from

$$
\begin{aligned}
& \left({\underset{\sim}{u}}_{0}^{k}, p_{0}^{k}\right)=I_{k-1}^{k}\left({\underset{\sim}{u-1}}_{k-1}^{*}, p_{k-1}^{*}\right), \\
& \left({\underset{\sim}{l}}_{l}^{k}, p_{l}^{k}\right)=M G\left(k,\left({\underset{\sim}{u-1}}_{l-1}^{k}, p_{l-1}^{k}\right),\left({\underset{\sim}{f}}_{k}, 0\right)\right), \quad 1 \leq l \leq r, \\
& \left({\underset{\sim}{u}}_{k}^{*}, p_{k}^{*}\right)=\left({\underset{\sim}{u}}_{r}^{k}, p_{r}^{k}\right),
\end{aligned}
$$

where $\underset{\sim}{f} \in \underset{\sim}{V} \underset{k}{\perp}$ satisfies

$$
\left({\underset{\sim}{f}}_{k}, \underset{\sim}{v}\right)_{\underset{\sim}{L^{2}(\Omega)}}=\frac{1}{2 \mu}\left[\int_{\Omega} \underset{\sim}{f} \cdot \underset{\sim}{v} d x+\left.\sum_{i=1}^{n} \int_{\Gamma_{i}} \underset{\sim}{g} \cdot \underset{\sim}{v}\right|_{\Gamma_{i}} d s\right]
$$

for all $\underset{\sim}{v} \in \underset{\sim}{V} \stackrel{\perp}{k}$, and $r$ is a positive integer independent of $k$ and $(\mu, \lambda)$. 
Since $I_{k-1}^{k}, I_{k}^{k-1}$, and $B_{k}$ are represented by sparse, banded matrices with $\mathscr{O}\left(n_{k}\right)$ nonzero entries, the total work of the full multigrid algorithm is therefore $\mathscr{O}\left(n_{k}\right)$. The proof is the same as the one in [1].

\section{Convergence analysis}

We follow the methodology of [1]. The details are given in the supplement to this paper. The first step is to discuss the convergence of the two-grid algorithm where the residual equation is solved exactly on the coarser grid. The final output of the two-grid algorithm for $(4.1)$ is therefore $\left(\underset{\sim}{y^{\sharp}}, z^{\sharp}\right):=\left(\underset{\sim}{y}, z_{m}\right)+$ $I_{k-1}^{k}\left(\stackrel{v}{\sim}^{\sharp}, q^{\sharp}\right)$, where

$$
\begin{aligned}
\left({\left.\stackrel{\sim}{v^{\sharp}}, q^{\sharp}\right)}^{\sharp}\right. & \left(B_{k-1}^{\perp}\right)^{-1}(\underset{\sim}{\bar{w}}, \bar{r}) \\
& =\left(B_{k-1}^{\perp}\right)^{-1} I_{k}^{k-1}\left((\underset{\sim}{w}, r)-B_{k}\left({\underset{\sim}{m}}_{m}^{y}, z_{m}\right)\right) \\
& =\left(B_{k-1}^{\perp}\right)^{-1} I_{k}^{k-1} B_{k}\left(\underset{\sim}{y}-\underset{\sim}{y}, z-z_{m}\right) .
\end{aligned}
$$

The following is the result for the two-grid algorithm.

Theorem (convergence of the two-grid algorithm). There exists a positive constant $C$ such that for $k>1$

$$
\left\|\left(\underset{\sim}{y}-{\underset{\sim}{y}}^{\sharp}, z-z^{\sharp}\right)\right\|_{0, k} \leq C m^{-1 / 2}\left\|\left(\underset{\sim}{y}-\underset{\sim}{y}, z-z_{0}\right)\right\|_{0, k},
$$

where $(\underset{\sim}{y}, z)$ solves $(4.1),\left(\underset{\sim}{y}, z_{0}\right)$ is the initial guess, and $\left(\underset{\sim}{y^{\sharp}}, z^{\sharp}\right)$ is the output of the two-grid algorithm. Therefore, for $m$ sufficiently large, the twogrid algorithm is a contraction with contraction number bounded away from one, independent of $k$.

The convergence theorem for the $k$ th-level iteration follows.

Theorem (convergence of the $k$ th-level iteration). There exists a positive constant $C$ such that when the kth-level iteration is applied to (4.1), we have

$$
\left\|(\underset{\sim}{y}, z)-M G\left(k,\left({\underset{\sim}{y}}_{0}^{y}, z_{0}\right),(\underset{\sim}{w}, r)\right)\right\|_{0, k} \leq C m^{-1 / 2}\left\|\left(\underset{\sim}{y}-\underset{\sim}{y}, z-z_{0}\right)\right\|_{0, k},
$$

provided that $m$ is chosen to be large enough. Therefore, for $m$ sufficiently large, the kth-level iteration is a contraction with contraction number bounded away from one, independent of $k$.

A perturbation argument then gives full multigrid convergence.

Theorem (full multigrid convergence). If $m$ is chosen large enough so that the $k$ th-level iteration is a contraction with respect to $\|\cdot\|_{0, k}$ and the parameter $r$ in the full multigrid algorithm is also chosen large enough, then

$$
\begin{gathered}
\left\|\underset{\sim}{u}-{\underset{\sim}{u}}_{k}^{*}\right\|_{\mathcal{\sim}^{2}(\Omega)}+h_{k}\left(\left\|\underset{\sim}{u}-\underset{\sim}{u_{k}^{*}}\right\|_{k}+\left\|p_{k}-p_{k}^{*}\right\|_{L^{2}(\Omega)}\right) \\
\leq C h_{k}^{2}\left\{\|f\|_{\mathcal{L}^{2}(\Omega)}+\sum_{i=1}^{n}\|\underset{\sim}{g}\|_{\sim_{\sim}^{1 / 2}\left(\Gamma_{i}\right)}\right\} .
\end{gathered}
$$

Here, $\left(\underset{\sim}{\sim}{ }_{k}^{*}, p_{k}^{*}\right)$ is the approximate solution of (1.26) obtained from the full multigrid algorithm. 


\section{A NUMERICAL EXPERIMENT}

The full multigrid algorithm described in $\S 4$ was applied to the pure traction boundary value problem (1.15) with $\mu=1 / 2$. The domain $\Omega$ is the unit square, the vertices are $S_{1}=(0,0), S_{2}=(1,0), S_{3}=(1,1)$ and $S_{4}=(0,1)$, and $\mathscr{T}_{0}$ consists of $\triangle S_{1} S_{2} S_{4}$ and $\Delta S_{2} S_{3} S_{4}$. The body force $\underset{\sim}{f}=\left(f_{1}, f_{2}\right)^{t}$ is given by

$$
\begin{aligned}
& f_{1}=-\pi^{2} \sin \pi x_{1} \sin \pi x_{2}+2 \pi^{2}\left(\frac{1}{\lambda}+1\right) \cos \pi x_{1} \sin \pi x_{2}, \\
& f_{2}=-\pi^{2} \cos \pi x_{1} \cos \pi x_{2}+2 \pi^{2}\left(\frac{1}{\lambda}+1\right) \sin \pi x_{1} \cos \pi x_{2},
\end{aligned}
$$

and the tractions are given by

$$
\begin{aligned}
& \underset{\sim}{g}=\left(-\frac{\pi}{\lambda} \cos \pi x_{1}, 0\right)^{t}, \quad \underset{\sim}{g}=\left(\pi \sin \pi x_{2},-\frac{\pi}{\lambda} \cos \pi x_{2}\right)^{t}, \\
& \underset{\sim}{g}=\left(-\frac{\pi}{\lambda} \cos \pi x_{1}, 0\right)^{t}, \quad \text { and } \quad \underset{\sim}{g}=\left(\pi \sin \pi x_{2},-\frac{\pi}{\lambda} \cos \pi x_{2}\right)^{t} .
\end{aligned}
$$

The exact solution $\underset{\sim}{u}=\left(u_{1}, u_{2}\right)^{t} \in \underset{\sim}{H_{\perp}^{2}}$ is

$$
\begin{aligned}
& u_{1}=\left(-\sin \pi x_{1}+\frac{1}{\lambda} \cos \pi x_{1}\right) \sin \pi x_{2}+\frac{4}{\pi^{2}}, \\
& u_{2}=\left(-\cos \pi x_{1}+\frac{1}{\lambda} \sin \pi x_{1}\right) \cos \pi x_{2} .
\end{aligned}
$$

In the following table, $\nu=\lambda / 2\left(\frac{1}{2}+\lambda\right)$ is the Poisson ratio, $h$ represents the lengths of the horizontal and vertical sides of the triangles in the triangulation, $r$ is the number of nested iterations in the full multigrid algorithm, and the numbers in the third, fifth, and seventh (respectively fourth, sixth, and eighth) columns represent the number $m$ of smoothing steps required to achieve an $L^{2}$ relative error of less that 5\% (respectively 3\%) in the displacement. It is observed that this algorithm fails to converge for this problem for $m \leq 4$.

\begin{tabular}{|c|c|c|c|c|c|c|c|}
\hline \multirow{2}{*}{$\nu$} & \multirow{2}{*}{$\nu$} & \multicolumn{2}{|c|}{$r=20$} & \multicolumn{2}{c|}{$r=30$} & \multicolumn{2}{c|}{$r=40$} \\
\cline { 2 - 8 } & & $m(5 \%)$ & $m(3 \%)$ & $m(5 \%)$ & $m(3 \%)$ & $m(5 \%)$ & $m(3 \%)$ \\
\hline \multirow{3}{*}{0.47619} & $(0.5)^{6}$ & 13 & 19 & 9 & 13 & 7 & 10 \\
& $(0.5)^{7}$ & 7 & 9 & 5 & 6 & 5 & 5 \\
& $(0.5)^{8}$ & 5 & 5 & 5 & 5 & 5 & 5 \\
\hline \multirow{3}{*}{0.49751} & $(0.5)^{6}$ & 20 & 27 & 15 & 19 & 12 & 15 \\
& $(0.5)^{7}$ & 11 & 14 & 8 & 10 & 6 & 8 \\
& $(0.5)^{8}$ & 6 & 8 & 5 & 5 & 5 & 5 \\
\hline \multirow{3}{*}{0.49975} & $(0.5)^{6}$ & 21 & 28 & 15 & 20 & 12 & 16 \\
& $(0.5)^{7}$ & 12 & 15 & 8 & 11 & 7 & 9 \\
& $(0.5)^{8}$ & 6 & 8 & 5 & 6 & 5 & 5 \\
\hline
\end{tabular}

\section{BIBLIOGRAPHY}

1. R.E. Bank and T. Dupont, An optimal order process for solving finite element equations, Math. Comp. 36 (1981), 35-51.

2. S.C. Brenner, A nonconforming multigrid method for the stationary Stokes equations, Math. Comp. 55 (1990), 411-437. 
3. S.C. Brenner and L.Y. Sung, Linear finite element methods for planar linear elasticity, Math. Comp. 59 (1992), 321-338.

4. P.G. Ciarlet, The finite element method for elliptic problems, North-Holland, Amsterdam, 1978.

5. M. Crouzeix and P.-A. Raviart, Conforming and nonconforming finite element methods for solving the stationary Stokes equations I, RAIRO Ser. Rouge 7 (1973), 33-75.

6. R.S. Falk, Nonconforming finite element methods for the equations of linear elasticity, Math. Comp. 57 (1991), 529-550.

7. P. Grisvard, Problèmes aux limites dans les polygones. Mode d'emploi, EDF Bulletin de la Direction des Etudes et Recherches, Series C, Mathématiques, Informatique 1 (1986), 21-59.

8. __ Singularités en elasticité, Arch. Rational Mech. Anal. 107 (1989), 157-180.

9. J. Nečas, Les méthodes directes en théorie des équations elliptiques, Masson, Paris, 1967.

10. J.A. Nitsche, On Korn's second inequality, RAIRO Anal. Numér. 15 (1981), 237-248.

11. I.D. Parsons and J.F. Hall, The multigrid method in solid mechanics: part I-algorithm description and behaviour, Internat. J. Numer. Methods Engrg. 29 (1990), 719-738.

12. R. Scott, Interpolated boundary conditions in the finite element method, SIAM J. Numer. Anal. 12 (1975), 404-427.

13. G. Strang and G. Fix, An analysis of the finite element method, Prentice-Hall, Englewood Cliffs, NJ, 1973.

14. J. M. Thomas, Sur l'analyse numérique des méthodes d'éléments finis hybrides et mixtes, Thèse d'Etat, Université Pierre et Marie Curie, Paris, 1977.

Department of Mathematics and Computer Science, Clarkson University, Potsdam, NEW YORK 13699-5815

Current address: Department of Mathematics, University of South Carolina, Columbia, South Carolina 29208

E-mail address: brenner@math. scarolina.edu 


\title{
Supplement to
}

\section{A NONCONFORMING MIXED MULTIGRID METHOD FOR THE PURE TRACTION PROBLEM IN PLANAR LINEAR ELASTICITY}

\author{
SUSANNE C. BRENNER
}

This supplement contains the proofs of the theorems stated in $\S 5$.

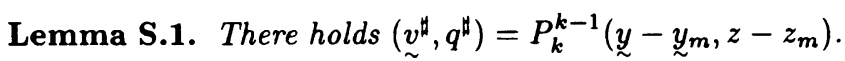

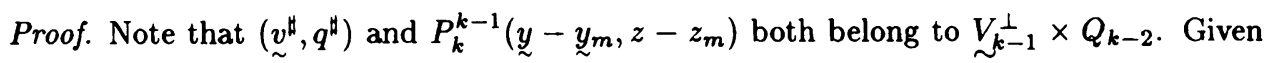
any $(\underset{\sim}{v}, q) \in \underset{\sim}{V_{k-1}^{\perp}} \times Q_{k-2}$, it follows from (3.4) and (3.11) that

$$
\begin{aligned}
\mathcal{B}_{k-1}\left(\left({\underset{\sim}{v}}^{\sharp}, q^{\sharp}\right),(\underset{\sim}{v}, q)\right) & =\left(B_{k-1}^{\perp}\left({\underset{\sim}{v}}^{\sharp}, q^{\sharp}\right),(\underset{\sim}{v}, q)\right)_{k-1} \\
& =\left(I_{k}^{k-1} B_{k}\left(\underset{\sim}{y}-\underset{\sim}{y_{m}}, z-z_{m}\right),(\underset{\sim}{v}, q)\right)_{k-1} \\
& =\left(B_{k}\left(\underset{\sim}{y}-{\underset{\sim}{y}}_{m}, z-z_{m}\right), I_{k-1}^{k}(\underset{\sim}{v}, q)\right)_{k} \\
& =\mathcal{B}_{k}\left(\left(\underset{\sim}{y}-\underset{\sim}{y}, z-z_{m}\right), I_{k-1}^{k}(\underset{\sim}{v}, q)\right) \\
& =\mathcal{B}_{k-1}\left(P_{k}^{k-1}\left(\underset{\sim}{y}-\underset{\sim}{y_{m}}, z-z_{m}\right),(\underset{\sim}{v}, q)\right) .
\end{aligned}
$$

The lemma now follows from Proposition 2.7.

Let the $k$ th-level relaxation operator $R_{k}$ be defined by

$$
R_{k}:=I-\frac{1}{\Lambda_{k}}\left(B_{k}^{\perp}\right)^{2} \text {. }
$$

(Recall that $\Lambda_{k}:=C h_{k}^{-4}$ dominates the spectral radius of $\left(B_{k}^{\perp}\right)^{2}$.)

Note that by the definition of the mesh-dependent norms we have

$$
\left\|R_{k}(\underset{\sim}{v}, q)\right\|_{s, k} \leq\|(\underset{\sim}{v}, q)\|_{s, k} \quad \forall(\underset{\sim}{v}, q) \in \underset{\sim}{V_{k}^{\perp}} \times Q_{k-1} \text { and } s \in \mathbb{R} .
$$

From the smoothing step (4.2), we obtain

$$
(\underset{\sim}{y}-\underbrace{}_{m}, z-z_{m})=R_{k}^{m}(\underset{\sim}{y}-\underbrace{}_{0}, z-z_{0}) \text {. }
$$

Combining Lemma S.1 and (S.3), we have the following relation between the initial error and the final error of the two-grid algorithm:

$$
(\underset{\sim}{y}-\underbrace{\sharp}_{\sim}, z-z^{\sharp})=\left(I-I_{k-1}^{k} P_{k}^{k-1}\right) R_{k}^{m}\left(\underset{\sim}{y}-{\underset{\sim}{y}}_{0}, z-z_{0}\right) .
$$

The effect of $R_{k}^{m}$ is measured by the following lemma on the smoothing property. 

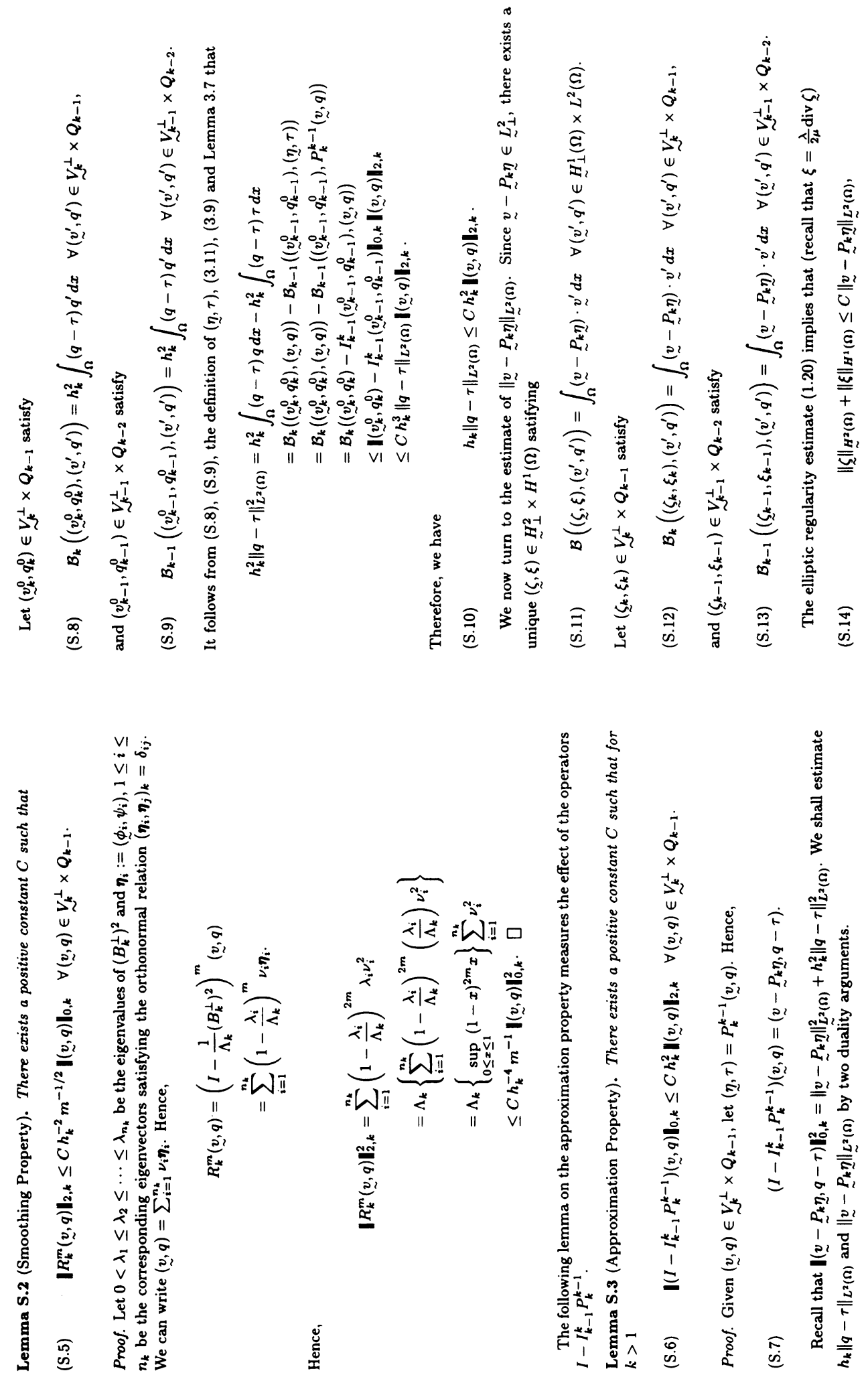

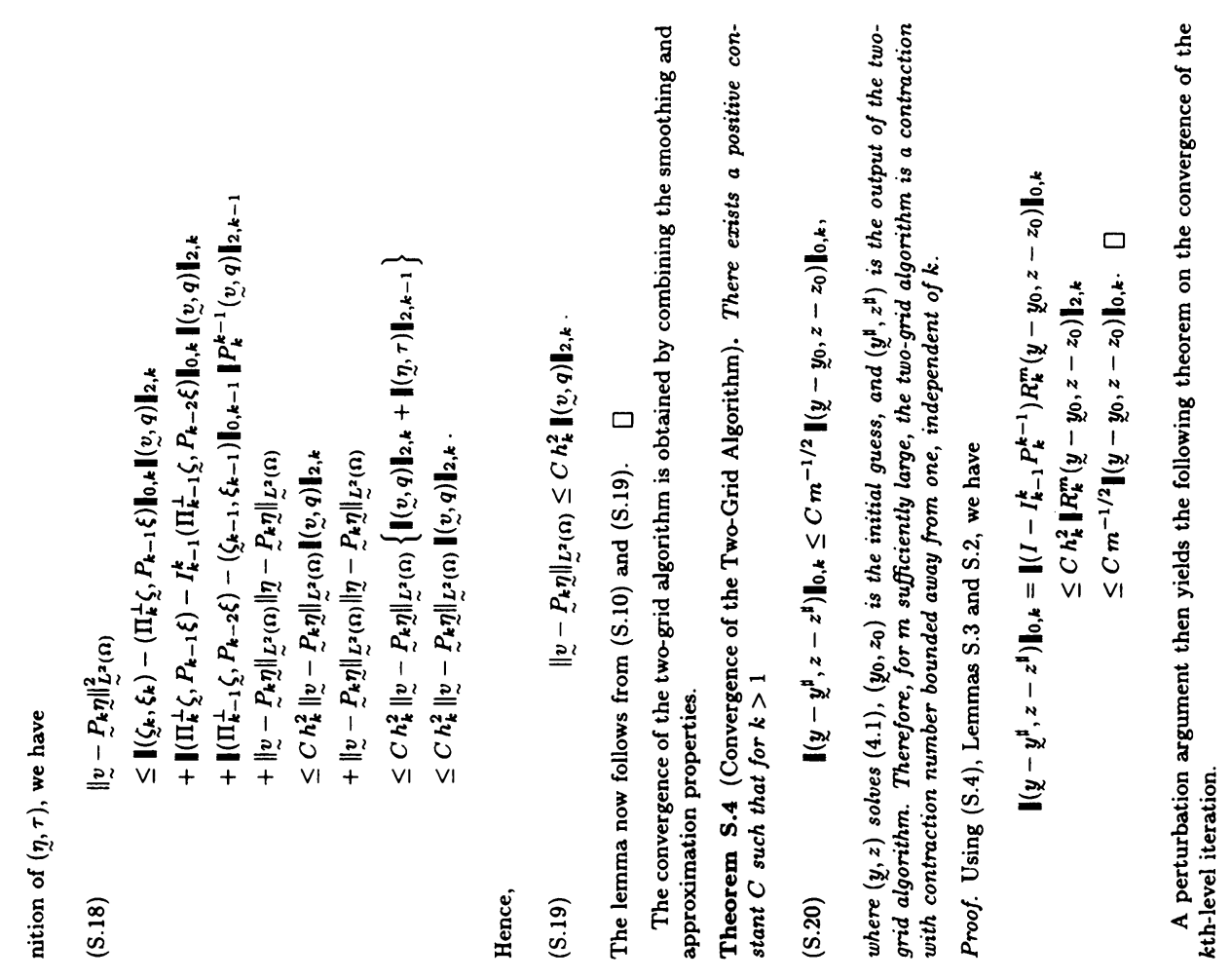

政

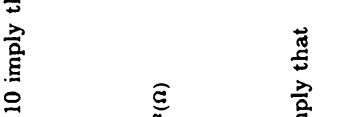

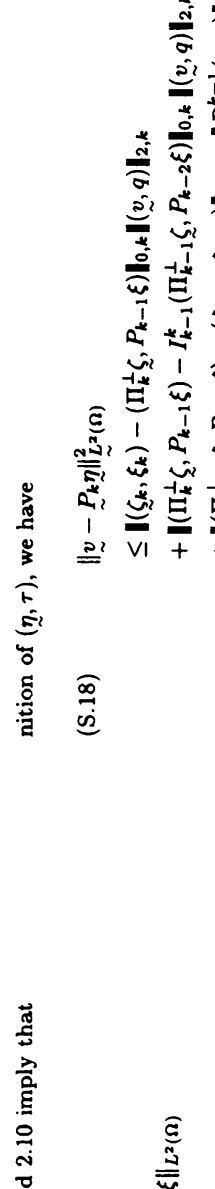

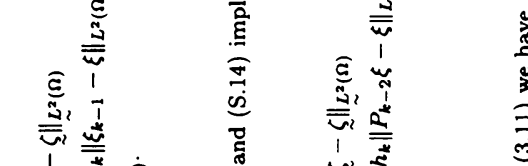

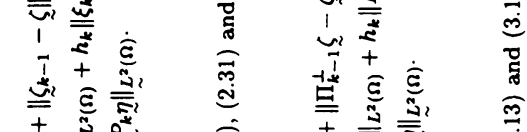

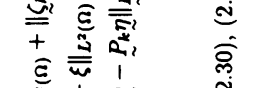

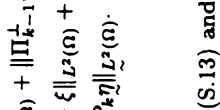

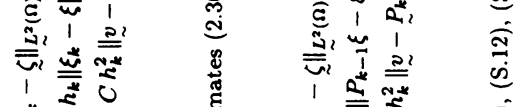

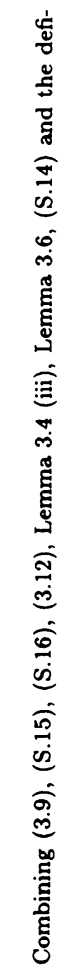



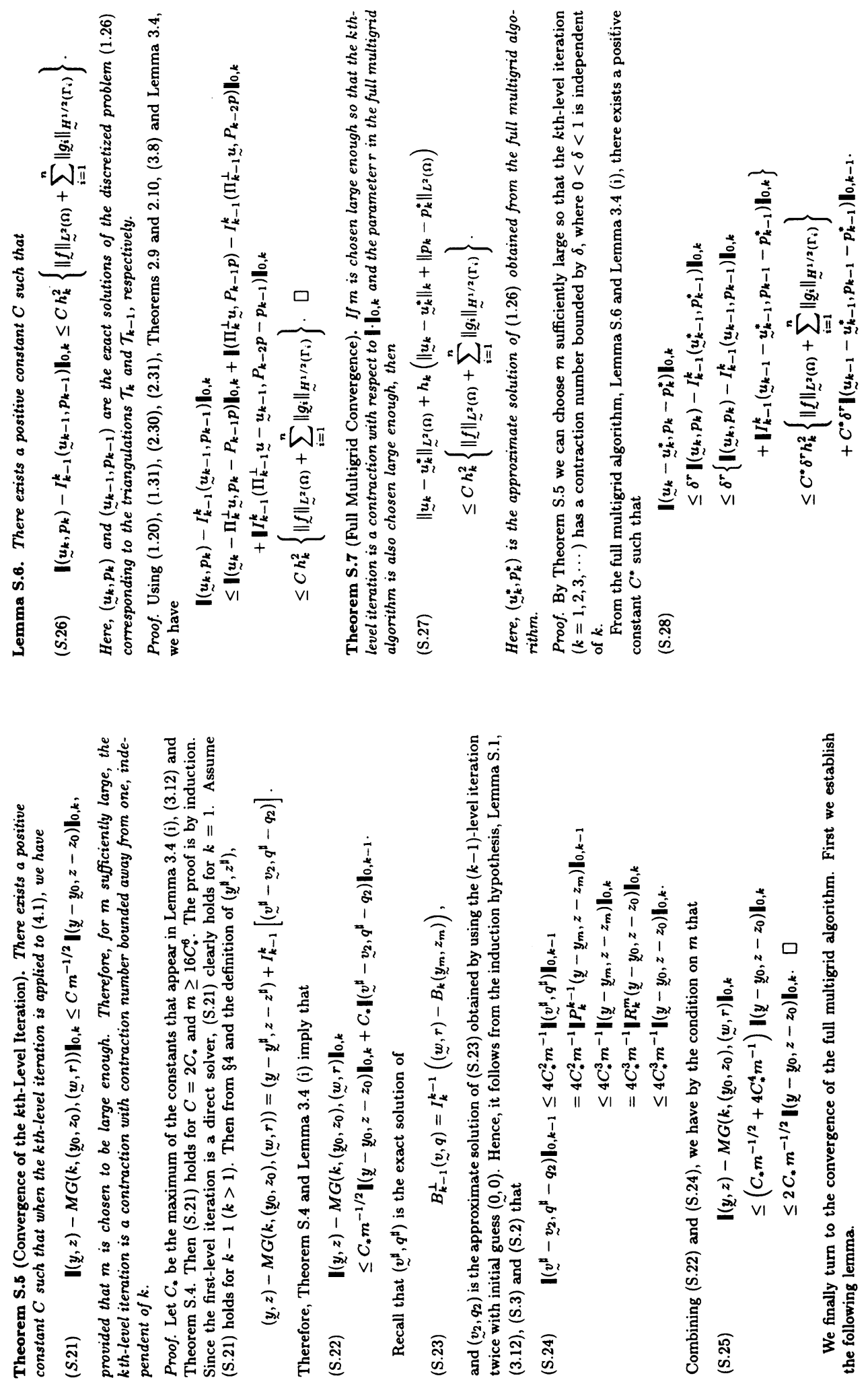


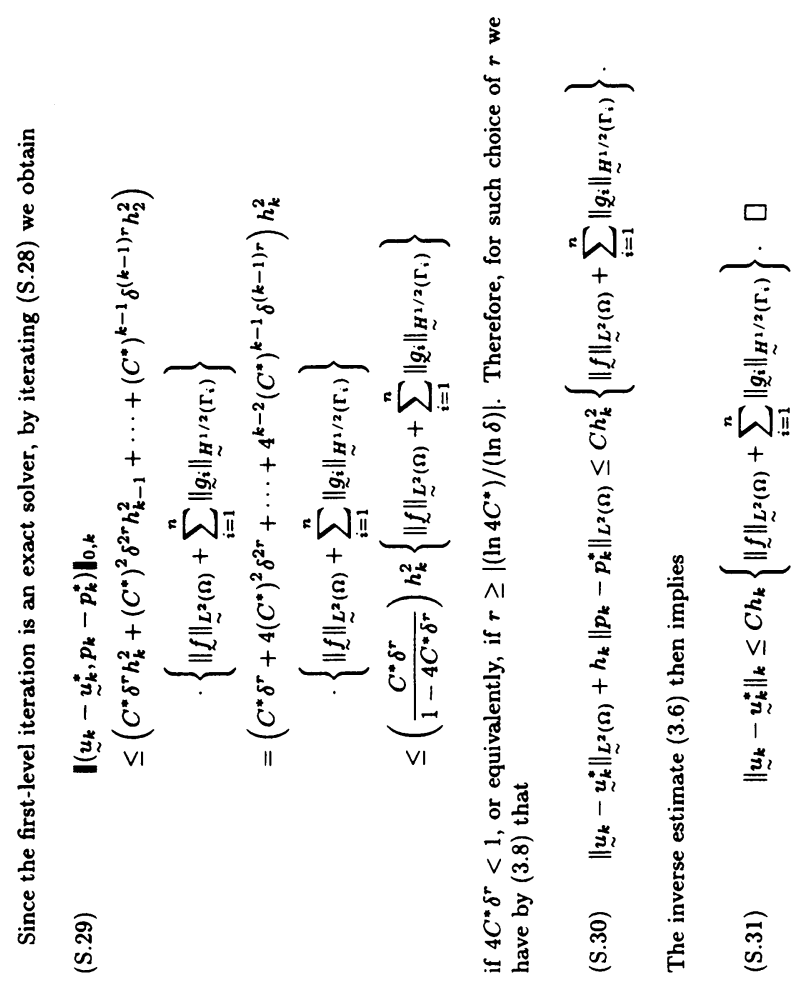

